\title{
Projected sensitivities of the LUX-ZEPLIN experiment to new physics via low-energy electron recoils
}

D. S. Akerib, ${ }^{1,2}$ A. K. Al Musalhi, ${ }^{3}$ S. K. Alsum, ${ }^{4}$ C. S. Amarasinghe,${ }^{5}$ A. Ames, ${ }^{1,2}$ T. J. Anderson, ${ }^{1,2}$ N. Angelides,${ }^{6}$ H. M. Araújo, ${ }^{7}$ J. E. Armstrong, ${ }^{8}$ M. Arthurs,${ }^{5}$ X. Bai, ${ }^{9}$ J. Balajthy, ${ }_{10}{ }^{6}$ S. Balashov, ${ }^{11}$ J. Bang, ${ }^{12}$ J. W. Bargemann, ${ }^{13}$ D. Bauer, ${ }^{7}$ A. Baxter, ${ }^{14}$ P. Beltrame, ${ }^{6}$ E. P. Bernard, ${ }^{15,16}$ A. Bernstein, ${ }^{17}$ A. Bhatti, ${ }^{8}$ A. Biekert, ${ }^{15,16}$ T. P. Biesiadzinski, ${ }^{1,2}$ H. J. Birch ${ }^{5}$ G. M. Blockinger, ${ }^{18}$ E. Bodnia, ${ }^{13}$ B. Boxer, ${ }^{10}$ C. A. J. Brew, ${ }^{11}$ P. Brás,${ }^{19}$ S. Burdin,${ }^{14}$ J. K. Busenitz, ${ }^{20}$ M. Buuck,${ }^{1,2}$ R. Cabrita, ${ }^{19}$ M. C. Carmona-Benitez, ${ }^{21}$ M. Cascella, ${ }^{6}$ C. Chan, ${ }^{12}$ N. I. Chott, ${ }^{9}$ A. Cole, ${ }^{16}$ M. V. Converse, ${ }^{22}$ A. Cottle, ${ }^{3}$ G. Cox,${ }^{21}$ O. Creaner, ${ }^{16}$ J. E. Cutter, ${ }^{10}$ C. E. Dahl,${ }^{23,24}$ L. de Viveiros, ${ }^{21}$ J. E. Y. Dobson, ${ }^{6}$ E. Druszkiewicz, ${ }^{22}$ S. R. Eriksen, ${ }^{25}$ A. Fan ${ }^{1,2}$ S. Fayer, ${ }^{7}$ N. M. Fearon, ${ }^{3}$ S. Fiorucci, ${ }^{16}$ H. Flaecher ${ }^{25}$ E. D. Fraser, ${ }^{14}$ T. Fruth, ${ }^{6}$ R. J. Gaitskell, ${ }^{12}$ J. Genovesi, ${ }^{9}$ C. Ghag, ${ }^{6}$ E. Gibson, ${ }^{3}$ S. Gokhale,${ }^{26}$ M. G. D. van der Grinten, ${ }^{11}$ C. B. Gwilliam, ${ }^{14}$ C. R. Hall, ${ }^{8}$ C. A. Hardy ${ }^{1,2}$ S. J. Haselschwardt, ${ }^{16}$ S. A. Hertel, ${ }^{27, *}$ M. Horn, ${ }^{28}$ D. Q. Huang, ${ }^{5}$ C. M. Ignarra, ${ }^{1}$ O. Jahangir, ${ }^{6}$ R. S. James, ${ }^{6}$ W. Ji, ${ }^{1,2}$ J. Johnson, ${ }^{10}$ A. C. Kaboth, ${ }^{29,11}$ A. C. Kamaha, ${ }^{18}$ K. Kamdin, ${ }^{16,15}$ K. Kazkaz, ${ }^{17}$ D. Khaitan, ${ }^{22}$ A. Khazov, ${ }^{11}$ I. Khurana, ${ }^{6}$ D. Kodroff, ${ }^{21}$ L. Korley, ${ }^{5}$ E. V. Korolkova, ${ }^{30}$ H. Kraus, ${ }^{3}$ S. Kravitz, ${ }^{16}$ L. Kreczko, ${ }^{25}$ B. Krikler, ${ }^{25}$ V. A. Kudryavtsev, ${ }^{30}$ E. A. Leason, ${ }^{31, \dagger}$ J. Lee, ${ }^{32}$ D. S. Leonard, ${ }^{32}$ K. T. Lesko, ${ }^{16}$ C. Levy, ${ }^{18} \mathrm{~J} . ~ L i^{32}{ }^{32}$ J. Liao, ${ }^{12}$ A. Lindote, ${ }^{19}$ R. Linehan, ${ }^{1}$

W. H. Lippincott, ${ }^{13,24}$ X. Liu, ${ }_{16}^{31}$ M. I. Lopes,${ }^{19}$ E. Lopez Asamar, ${ }^{19}$ B. López Paredes, ${ }^{7}$ W. Lorenzon, ${ }^{5}$ S. Luitz, ${ }^{1}$ P. A. Majewski, ${ }_{11}^{11}$ A. Manalaysay, ${ }^{16}$ L. Manenti, ${ }^{6}$ R. L. Mannino, ${ }^{4}$ N. Marangou ${ }^{7}$ M. E. McCarthy, ${ }^{22}$ D. N. McKinsey, ${ }^{15,16}$ J. McLaughlin, ${ }^{23}$ E. H. Miller, ${ }^{1,2}$ E. Mizrachi, ${ }^{17,8}$ A. Monte, ${ }^{13,24}$ M. E. Monzani, ${ }^{1,2}$ J. A. Morad, ${ }^{10}$ J. D. Morales Mendoza, ${ }^{1,2}$ E. Morrison, ${ }^{9}$ B. J. Mount, ${ }^{33}$ A. St. J. Murphy, ${ }^{31}$ D. Naim, ${ }^{10}$ A. Naylor, ${ }^{30}$ C. Nedlik,${ }^{27}$ H. N. Nelson, ${ }^{13}$ F. Neves, ${ }^{19}$ J. A. Nikoleyczik, ${ }^{4}$ A. Nilima, ${ }^{31,5}$ A. Nguyen, ${ }^{31}$ I. Olcina, ${ }^{15,16}$ K. C. Oliver-Mallory, ${ }^{7}$ S. Pal, ${ }^{19}$ K. J. Palladino, ${ }^{3,4}$ J. Palmer, ${ }^{29}$ S. Patton, ${ }^{16}$ N. Parveen,${ }^{18}$ E. K. Pease, ${ }^{16}$ B. Penning, ${ }^{5}$ G. Pereira, ${ }^{19}$ A. Piepke, ${ }^{20}$ Y. Qie, ${ }^{22}$ J. Reichenbacher, ${ }^{9}$ C. A. Rhyne, ${ }^{12}$ A. Richards, ${ }^{7}$ Q. Riffard, ${ }^{15,16}$ G. R. C. Rischbieter, ${ }^{18}$ R. Rosero, ${ }^{26}$ P. Rossiter, ${ }^{30}$ D. Santone, ${ }^{29}$ A. B. M. R. Sazzad, ${ }^{20}$ R. W. Schnee, ${ }^{9}$ P. R. Scovell, ${ }^{11}$ S. Shaw ${ }^{13}$ T. A. Shutt, ${ }^{1,2}$ J. J. Silk, ${ }^{8}$ C. Silva ${ }_{19}$ R. Smith, ${ }^{15,16}$ M. Solmaz, ${ }^{13}$ V. N. Solovov, ${ }^{19}$ P. Sorensen, ${ }^{16}$ J. Soria, ${ }^{15}$ I. Stancu, ${ }^{20}$ A. Stevens, ${ }^{3}$ K. Stifter, ${ }^{1,2}$ B. Suerfu, ${ }^{15,16}$ T. J. Sumner, ${ }^{7}$ N. Swanson, ${ }^{12}$ M. Szydagis ${ }^{18}$ W. C. Taylor, ${ }^{12}$ R. Taylor, ${ }^{7}$ D. J. Temples, ${ }^{23}$ P. A. Terman, ${ }^{34}$ D. R. Tiedt, ${ }^{28}$ M. Timalsina, ${ }^{9}$ W. H. To ${ }^{1,2}$ D. R. Tovey ${ }^{30}{ }^{30}$ M. Tripathi, ${ }^{10}$ D. R. Tronstad, ${ }^{9}$ W. Turner, ${ }^{14}$ U. Utku, ${ }^{6}$ A. Vaitkus, ${ }^{12}$ B. Wang, ${ }^{20}$ J. J. Wang, ${ }^{5}$ W. Wang, ${ }^{27,4}$ J. R. Watson, ${ }^{15,16}$ R. C. Webb, ${ }^{34}$ R. G. White, ${ }^{1,2}$ T. J. Whitis, ${ }^{13,1}$ M. Williams, ${ }^{5}$ F. L. H. Wolfs, ${ }^{22}$ D. Woodward, ${ }^{21}$ C. J. Wright, ${ }^{25}$ X. Xiang, ${ }^{12}$ J. Xu, ${ }^{17} \mathrm{M}$. Yeh, ${ }^{26}$ and P. Zarzhitsky ${ }^{20}$

${ }^{1}$ SLAC National Accelerator Laboratory, Menlo Park, California 94025-7015, USA

${ }^{2}$ Kavli Institute for Particle Astrophysics and Cosmology, Stanford University, Stanford, California 94305-4085, USA

${ }^{3}$ University of Oxford, Department of Physics, Oxford OX1 3RH, United Kingdom

${ }^{4}$ University of Wisconsin-Madison, Department of Physics, Madison, Wisconsin 53706-1390, USA

${ }^{5}$ University of Michigan, Randall Laboratory of Physics, Ann Arbor, Michigan 48109-1040, USA

${ }^{6}$ University College London (UCL), Department of Physics and Astronomy, London WCIE 6BT, United Kingdom

${ }^{7}$ Imperial College London, Physics Department, Blackett Laboratory, London SW7 2AZ, United Kingdom

${ }^{8}$ University of Maryland, Department of Physics, College Park, Maryland 20742-4111, USA

${ }^{9}$ South Dakota School of Mines and Technology, Rapid City, South Dakota 57701-3901, USA

${ }^{10}$ University of California, Davis, Department of Physics, Davis, California 95616-5270, USA

${ }^{11}$ STFC Rutherford Appleton Laboratory (RAL), Didcot OX11 OQX, United Kingdom

${ }^{12}$ Brown University, Department of Physics, Providence, Rhode Island 02912-9037, USA

${ }^{13}$ University of California, Santa Barbara, Department of Physics, Santa Barbara, California 93106-9530, USA

${ }^{14}$ University of Liverpool, Department of Physics, Liverpool L69 7ZE, United Kingdom

${ }^{15}$ University of California, Berkeley, Department of Physics, Berkeley, California 94720-7300, USA

${ }^{16}$ Lawrence Berkeley National Laboratory (LBNL), Berkeley, California 94720-8099, USA

${ }^{17}$ Lawrence Livermore National Laboratory (LLNL), Livermore, California 94550-9698, USA

${ }^{18}$ University at Albany (SUNY), Department of Physics, Albany, New York 12222-0100, USA

${ }^{19}$ Laboratório de Instrumentação e Física Experimental de Partículas (LIP), University of Coimbra, P-3004 516 Coimbra, Portugal

${ }^{20}$ University of Alabama, Department of Physics and Astronomy, Tuscaloosa, Alabama 34587-0324, USA

${ }^{21}$ Pennsylvania State University, Department of Physics University Park, Pennsylvania 16802-6300, USA

${ }^{22}$ University of Rochester, Department of Physics and Astronomy, Rochester, New York 14627-0171, USA

${ }^{23}$ Northwestern University, Department of Physics \& Astronomy, Evanston, Illinois 60208-3112, USA 


\author{
${ }^{24}$ Fermi National Accelerator Laboratory (FNAL), Batavia, Illinois 60510-5011, USA \\ ${ }^{25}$ University of Bristol, H.H. Wills Physics Laboratory, Bristol BS8 1TL, United Kingdom \\ ${ }^{26}$ Brookhaven National Laboratory (BNL), Upton, New York 11973-5000, USA \\ ${ }^{27}$ University of Massachusetts, Department of Physics, Amherst, Massachusetts 01003-9337, USA \\ ${ }^{28}$ South Dakota Science and Technology Authority (SDSTA), Sanford Underground Research Facility, \\ Lead, South Dakota 57754-1700, USA \\ ${ }^{29}$ Royal Holloway, University of London, Department of Physics, Egham TW20 OEX, United Kingdom \\ ${ }^{30}$ University of Sheffield, Department of Physics and Astronomy, Sheffield S3 7RH, United Kingdom \\ ${ }^{31}$ SUPA, School of Physics and Astronomy, University of Edinburgh, \\ Edinburgh EH9 3FD, United Kingdom \\ ${ }^{32}$ IBS Center for Underground Physics (CUP), 34126, Yuseong-gu, Daejeon, Korea \\ ${ }^{33}$ Black Hills State University, School of Natural Sciences, Spearfish, South Dakota 57799-0002, USA \\ ${ }^{34}$ Texas A\&M University, Department of Physics and Astronomy, College Station, Texas 77843-4242, USA
}

(Received 18 May 2021; accepted 22 September 2021; published 23 November 2021)

LUX-ZEPLIN is a dark matter detector expected to obtain world-leading sensitivity to weaklyinteracting massive particles interacting via nuclear recoils with a $\sim 7$-tonne xenon target mass. This paper presents sensitivity projections to several low-energy signals of the complementary electron recoil signal type: 1) an effective neutrino magnetic moment, and 2) an effective neutrino millicharge, both for $p p$-chain solar neutrinos, 3) an axion flux generated by the Sun, 4) axionlike particles forming the Galactic dark matter, 5) hidden photons, 6) mirror dark matter, and 7) leptophilic dark matter. World-leading sensitivities are expected in each case, a result of the large $5.6 \mathrm{t} 1000 \mathrm{~d}$ exposure and low expected rate of electron-recoil backgrounds in the $<100 \mathrm{keV}$ energy regime. A consistent signal generation, background model and profile-likelihood analysis framework is used throughout.

DOI: $10.1103 /$ PhysRevD.104.092009

\section{INTRODUCTION}

LUX-ZEPLIN (LZ) is a liquid xenon (LXe) timeprojection chamber (TPC) currently being commissioned at the Sanford Underground Research Facility (SURF) in the USA [1]. It is the latest in a series of increasingly large LXe TPCs optimized for sensitivity to rare keV-scale nuclear recoil signatures indicative of weakly-interacting massive particle (WIMP) dark matter [2-10]. These detectors achieve extremely low background rates thanks to the very high purity of the LXe material itself and the selfshielding of the LXe, which inhibits the penetration of external radiation into the central fiducial LXe volume.

While the design goal of these dark matter detectors is sensitivity to nuclear recoils, several aspects of that optimization - a keV-scale energy threshold, large size, and low background-together provide complementary

\footnotetext{
* Corresponding author. shertel@umass.edu

Corresponding author. e.a.leason@sms.ed.ac.uk

Corresponding author. s1642680@sms.ed.ac.uk

Published by the American Physical Society under the terms of the Creative Commons Attribution 4.0 International license. Further distribution of this work must maintain attribution to the author(s) and the published article's title, journal citation, and DOI. Funded by SCOAP.
}

sensitivity to low-energy, low-rate processes of electron recoil (ER) type. In this paper we present sensitivity studies for several novel processes that would produce low-rate ER signatures with energy depositions smaller than $100 \mathrm{keV}$. Section II describes the essential aspects of the LZ instrument. Section III gives a brief description of the analysis framework used. Section IV presents the background model with particular emphasis on low-energy ER backgrounds. In Sec. V, the signal models for each of the different novel physics processes are presented. Section VI briefly presents the profile likelihood ratio (PLR) statistical method used to determine the sensitivity reach of LZ to each of the possible signals. Results are presented in Sec. VII. For each signal model, a projection for $90 \%$ C.L. exclusion sensitivity is presented. For selected signal models, projected sensitivity for $3 \sigma$ evidence is also presented. Conclusions are presented in Sec. VIII.

\section{DESCRIPTION OF THE EXPERIMENT}

The LZ experiment is located at a depth of $1480 \mathrm{~m}$ (4300 m water equivalent), which reduces the cosmic-ray muon flux by a factor of $\sim 10^{6}$ [11]. The LXe detector is surrounded by an instrumented water tank that shields against backgrounds, including gamma rays originating in the surrounding rock. The LXe cryostat is immediately surrounded by an active veto layer of Gd-loaded liquid scintillator (the outer detector), primarily for tagging nuclear recoils in the TPC, but also effective at further 
reducing ER backgrounds. The LXe is contained within a double-walled cylindrical vessel fabricated from low background titanium [12]. Surrounding the TPC on the sides and bottom is a $\sim 2$-tonne layer of optically-isolated LXe (the LXe skin) that is separately instrumented and serves as an active veto to further reduce backgrounds in the central volume. The central TPC volume includes a liquid/gas interface near the top of the volume, such that a thin layer of gaseous xenon is maintained for signal generation purposes. A vertical electric field, anticipated to be $\sim 310 \mathrm{~V} / \mathrm{cm}$ in the LXe, is applied using an anode placed within the gas layer and a cathode towards the bottom of the liquid. Incident radiation scattering within the LXe generates electron and/or nuclear recoils, in turn leading to electronic excitations and ionizations. Deexcitations produce primary scintillation (denoted 'S1'), while ionization electrons that escape recombination are drifted by the electric field to the liquid surface. A gate grid is located just under the liquid surface to assist with extraction of these electrons to the gas phase, from where a delayed second signal ('S2') of electroluminescence is generated. The electroluminescence gain in the gas region allows observation of single electrons emitted from the liquid surface. In analyses requiring an S1 signal (as is the case in this manuscript), it is the $\mathrm{S} 1$ signal that sets the energy threshold. The simulations of this work project the $10 \%$, $50 \%$, and $90 \%$ detection efficiency thresholds to be 1.04 , 1.45 , and $2.14 \mathrm{keV}$ respectively.

Arrays of high quantum efficiency, vacuum ultraviolet sensitive, low radioactivity photomultiplier tubes (PMTs), Hamamatsu model R11410-20, are placed both above (253 PMTs) and below (241 PMTs) the TPC volume. The S1 signal is relatively small, while the delayed secondary $\mathrm{S} 2$ is larger, with the signal amplitude ratio S2/S1 typically larger for ER interactions and smaller for NR interactions. The horizontal location of an energy deposition may be reconstructed from the distribution of S2 signal amplitudes in the upper PMT array, and the vertical location can be inferred from the time delay between the S1 and S2. A large fraction of energy depositions originate from trace radioactivity of the surrounding materials, and thus are observed near the edges of the LXe volume. The precise event position information given by the TPC configuration enables the definition in off-line analysis of a central fiducial volume of extremely low background. The LZ TPC surrounds a LXe mass of 7.0 tonnes; the central fiducial volume is taken as 5.6 tonnes. The low-energy ER response of the detector will be characterized using radioactive sources individually injected into the fiducial volume, including the monoenergetic sources ${ }^{83 \mathrm{~m}} \mathrm{Kr}(41 \mathrm{keV})$ and ${ }^{131 \mathrm{~m}} \mathrm{Xe}(164 \mathrm{keV})$ and the beta decaying sources ${ }^{3} \mathrm{H}$ and ${ }^{14} \mathrm{C}$ (in the form of ${ }^{3} \mathrm{H}$-labeled and ${ }^{14} \mathrm{C}$-labeled $\mathrm{CH}_{4}$ ). The use of labeled $\mathrm{CH}_{4}$ sources requires specific precautions to avoid introducing trace amounts of ${ }^{3} \mathrm{H}$ and ${ }^{14} \mathrm{C}$ in other chemical forms (e.g., other hydrocarbons or water). The varied experiences of recent
LXe experiments [13-15] have emphasized the need for a specific $\mathrm{CH}_{4}$ purifier. Even with this precaution, lingering ${ }^{3} \mathrm{H}$ or ${ }^{14} \mathrm{C}$ contamination will be searched for given its potential effect on the searches described here. External gamma-ray sources and neutron sources will also be used. Further details of the LZ apparatus may be found in Refs. [16] and [17].

Previous papers have described expected LZ sensitivities to signatures of new physics: WIMP sensitivity is described in [18] and [19], and sensitivity to the neutrinoless double beta decay of ${ }^{136} \mathrm{Xe}$ is described in [20]. The background models in those studies and this paper are built by first conducting a campaign of material assays [21] and then simulating the effects of those trace amounts of radioactivity in the LZ geometry [11]. Also simulated is the production and collection of S1 and S2 light signals. The observed signals are measured in units of photons detected (phd), an observable that accounts for the occurrence of double photoelectron emission from PMTs [22,23]. The average fraction of $\mathrm{S} 1$ scintillation photons that are detected is denoted $g_{1}$ and is predicted by simulation to be $0.119 \mathrm{phd} /$ photon, while the average number of photoelectrons generated by an ionization electron extracted from the liquid surface is denoted $g_{2}$ and is predicted to be $79.2 \mathrm{phd} /$ electron. While single photoelectrons produce a signal clearly separable from electronic noise, an anticipated $\mathrm{kHz}$-scale dark rate of single electron emission (within the PMTs) results in a three-fold S1 coincidence requirement, meaning a 3 phd $\mathrm{S} 1$ threshold. (This coincidence requirement can be reduced as in [24] and [19], but this technique is not taken advantage of by this analysis.) All detector response characteristics in this analysis match previous LZ sensitivity papers, see Refs. [18,20].

\section{ANALYSIS FRAMEWORK}

The LZ simulation chain [11] was used to generate the expected distributions of detector observables for radiation emitted from each of the background components and for each signal model. For those background components which are either nonuniform in spatial distribution or possibly registered as multiple scatters, we begin with an in-house software package that employs GEANT4 [25] to track particles as they propagate through the detector. Several of the physics routines have been modified to better model interactions with xenon and gadoliniumloaded scintillators. Energy deposits, timing, and spatial information for the interaction are recorded and the photonlevel signal response for each PMT generated. The timing and spatial information are used to enable the application of data selection cuts to the simulated data. Scatters are accepted as single-scatter events if there is no coincident energy deposit in the active skin or outer detector volumes (given nominal thresholds) and if their several energy deposits in the LXe are of small spatial extent. Quantitatively, this spatial extent requirement is defined as 
having an energy-weighted standard deviation $<3 \mathrm{~cm}$ in the radial direction and $<0.2 \mathrm{~cm}$ in the vertical direction, imitating the effect of selection cuts based on S2 light distribution and pulse shape.

For those event types which are both uniform in space and always appear as single scatters, the GEANT4 framework is not necessary, and we instead use theoretical energy spectra generated analytically, giving the expected number of events per unit mass and time, which can then be scaled according to exposure. This simpler framework is used for each of the signal models described in Sec. V, along with the simplest background components $\left({ }^{136} \mathrm{Xe}\right.$ decay, ${ }^{124} \mathrm{Xe}$ decay, and both ER and NR neutrino scatters).

After these background and signal energy spectra are compiled, each is then used as input to the Noble Element Simulation Technique (NEST) software [26,27], where ER and NR energy deposits are translated to the detector observables S1 and S2. The position dependence of signal collection efficiencies is included in that translation to $\mathrm{S} 1$ and S2. Finally, that position dependence is removed by applying position-dependent 'corrections' as planned to actual data, to form the final analysis quantities S1c and S2c. These simulations of signal production and collection are based on detector response parameters specific to the LZ detector (including, for example, $g_{1}, g_{2}$, and electricfield amplitude).

After signals are simulated, selections are applied mimicking those selections that will be applied to real data. Valid events must have an S1 in which at least three PMTs register at least a single photoelectron, while the uncorrected S2 signal is required to be greater than 415 phd, equivalent to $\approx$ five electrons emitted from the liquid surface, ensuring that the radial position of the scatter is adequately reconstructed. Fiducial volume cuts $r<68.8 \mathrm{~cm}$ from the center and $1.5 \mathrm{~cm}<z<132.1 \mathrm{~cm}$ above the cathode are applied to define the 5.6-tonne fiducial mass, consistent with [18].

\section{BACKGROUNDS}

LZ sensitivity to rare ER signals is limited by a combination of radiogenic and cosmogenic backgrounds. With limited exceptions, the backgrounds of this manuscript are modeled as in [18], and for that reason we do not repeat a detailed discussion here, describing only changes relative to that work. The first change is a more accurate treatment of the atomic binding effects in the solar-neutrino electron-scattering spectrum [28]. While $p p$ and ${ }^{7} \mathrm{Be}$ neutrinos dominate the neutrino-scattering rate, there are additional contributions from the $\mathrm{CNO},{ }^{8} \mathrm{~B}$, hep and pep mechanisms. Scattering rates are taken from the relativistic random phase approximation (RRPA) [29], used directly up to $30 \mathrm{keV}$. Above $30 \mathrm{keV}$, a simpler method based on the stepping approximation [30] is used, scaled to agree with RRPA at $30 \mathrm{keV}$.
The second change is the inclusion of two-neutrino double-electron capture (DEC) of ${ }^{124} \mathrm{Xe}$ as a background contribution. We include the monoenergetic peaks from the three most frequent $K$ - and $L$-shell capture combinations: $64.3 \mathrm{keV}$ ( $K K$-shell capture, branching fraction $76.6 \%), 36.7 \mathrm{keV}$ (KL-shell capture, $23 \%$ ), and $9.8 \mathrm{keV}$ ( $L L$-shell capture, $1.7 \%)$. The decay rate of $(1.8 \pm 0.5 \pm$ $0.1) \times 10^{22}$ years is informed by the XENON1T experiment's recently-reported evidence for $K K$-shell capture [31]. Neutron capture on ${ }^{124} \mathrm{Xe}$ (mostly on the unshielded xenon outside the water tank) will result in constant lowlevel production of ${ }^{125} \mathrm{I}$, with decay rate in the TPC highly dependent on iodine's specific purification timescale. Conservatively, assuming a 10 day purification half-life as a rough scale (significantly slower than the timescale of Xe recirculation in LZ), we anticipate $\sim 100{ }^{125}$ I decays in the 5.6 tonne 1000 day exposure. Because this number is small in comparison to the ${ }^{124} \mathrm{Xe} K K$-shell background (2,527 counts expected), and nearly degenerate in energy $(67.3 \mathrm{keV}),{ }^{125} \mathrm{I}$ is not included in the background model of this analysis.

A third change is the introduction of two unexpected but possible background components. Although this is a broad class, we introduce two such components which have a relevant rate in the few-keV regime where XENON1T recently reported evidence of an excess $[32-34] ;{ }^{37} \mathrm{Ar}$ (treated as a monoenergetic peak at $2.8 \mathrm{keV}$ ) and ${ }^{3} \mathrm{H}$ (a beta decay with a broad peak at $\sim 3 \mathrm{keV}$ and endpoint of $18.6 \mathrm{keV}$ ). These two backgrounds are expected to have negligible long-term contribution and are therefore not included in the projections of exclusion sensitivity. However, if an excess is seen that is consistent with both new physics and such a background, it would be difficult to rule out the background hypothesis and claim evidence or discovery. Both radioisotopes can be formed via spallation, for example, and there may be an unknown production path in detector materials. Indeed, our own calculations suggest cosmogenically produced ${ }^{37} \mathrm{Ar}$ will be present at some level in the earliest LZ data [35]. We therefore conservatively include such unexpected but possible backgrounds when quantifying sensitivity to evidence of signal (the exclusion of the background-only hypothesis). The method of this inclusion is described in Sec VI.

Recently, several authors (see Refs. [32,36]) have offered improved descriptions of the spectral shape of the ${ }^{214} \mathrm{~Pb}$, ${ }^{212} \mathrm{~Pb}$, and ${ }^{85} \mathrm{Kr}$ beta decay spectra, particularly at low energies. These refinements are not included in the present work, but the effect of such a reduction in the rate of these background components is discussed in Sec. VII B. As in [18], the two-neutrino double beta decay $(\nu \nu \beta \beta)$ spectrum of ${ }^{136} \mathrm{Xe}$ follows [37] without uncertainty.

Figure 1 shows the predicted ER background event rates, both in terms of a rate (counts/[kg day $\mathrm{keV}]$ ), and in terms of counts (per keV) for the full LZ exposure of 1000 days. Here we plot the spectra of true deposited energy, meaning 


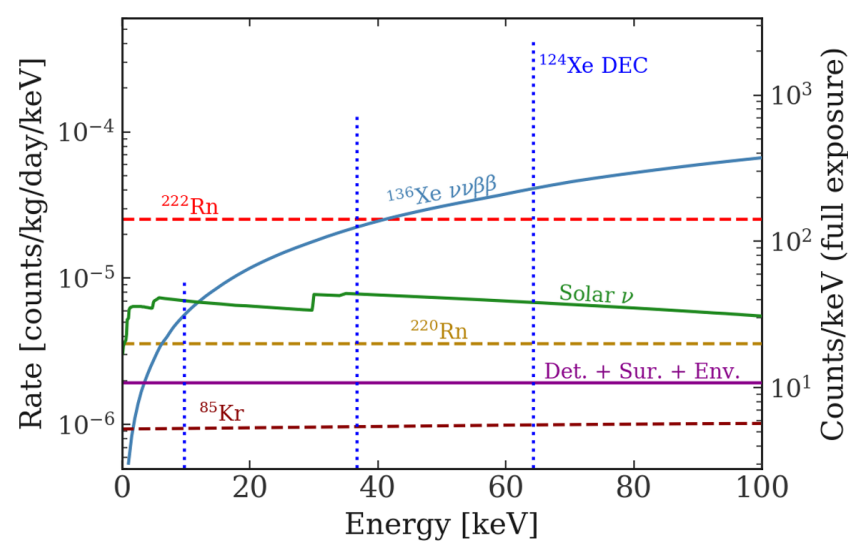

FIG. 1. The ER backgrounds expected in LZ, after application of veto anticoincidence, single-scatter, and fiducial-volume selections. The left axis indicates rate in standard units; the right axis indicates counts per $\mathrm{keV}$ in the anticipated 5.6 tonne 1000 day LZ exposure. This is true energy, where effects of detector resolution and threshold are not included. The three dashed curves indicate the three species that are present as contaminants within the LXe, for which some uncertainty exists on their final concentrations. The curve labeled 'Det.+Sur.+Env.' identifies contributions from the Compton scattering of $\gamma$-rays emitted from the bulk and surfaces of detector components, and from the laboratory and rock environment. Three monoenergetic peaks from ${ }^{124} \mathrm{Xe}$ double electron capture decay are indicated as lines (for which the units are counts/kg/day and counts on the left and right axes, respectively).

detector resolution and threshold are not included. NR background rates are dominated by coherent elastic scattering of ${ }^{8} \mathrm{~B}$ neutrinos, and while NR backgrounds are included in the sensitivity projections of this manuscript, they are not shown in Fig. 1 because their effect on ER

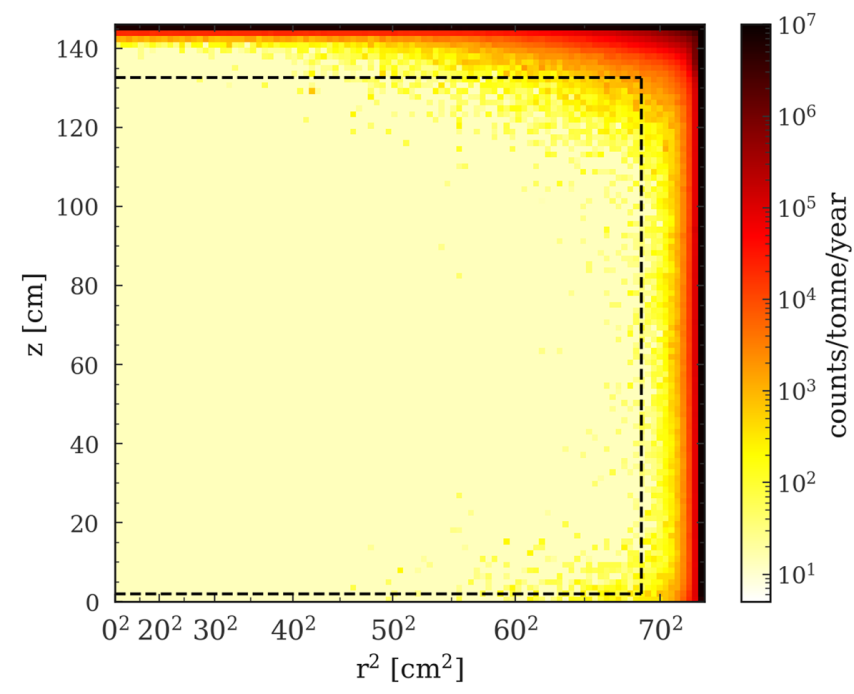

FIG. 2. Spatial distribution of all ER backgrounds passing single scatter and veto anti-coincidence selection criteria, with energy less than $100 \mathrm{keV}$. The 5.6 tonne fiducial region is indicated by the dashed black line. sensitivities is negligible. Quantitatively, over the lifetime of LZ, we expect $<1$ NR appearing within the $3 \sigma(99.7 \%)$ contours of the ER region of the $\{\mathrm{S} 1 \mathrm{c}, \log \mathrm{S} 2 \mathrm{c}\}$ plane. The total background rate is dominated below $\sim 40 \mathrm{keV}$ by the decay of ${ }^{222} \mathrm{Rn}$ progeny (predominantly the ground state beta decay of ${ }^{214} \mathrm{~Pb}$ ), and is dominated at higher energies by the $\nu \nu \beta \beta$ decay of ${ }^{136} \mathrm{Xe}$. All of these background components are uniform in rate throughout the TPC with the exception of the component labeled 'Detector + Surface + Environment'. This component leads to spatial dependence in the total background rate and to the definition of the fiducial volume selection, illustrated in Fig. 2. In both Figs. 1 and 2 , veto and single-scatter selections have been applied.

\section{SIGNAL MODELS}

In this section we present seven examples of novel physics processes that would produce low-energy ER signals in LZ. For each new physics process, the theoretical motivation is briefly recapped and the expected deposited energy spectra and overall interaction rate are described as a function of the relevant cross section, coupling constant, or other physical parameter. As described in Sec. III, these true-energy spectra are then translated into the experimental observables of S1c and S2c, before testing their observability given the expected backgrounds. The range of models chosen includes a variety of spectral shapes, from monoenergetic signatures to gently sloping spectra.

Figure 3 describes the predicted spectra for the various signals considered. The spectra of recoil energy (the true energy deposited) are shown by dashed curves in each plot. As described in Sec. III, a NEST simulation then takes those true energies as inputs to generate the detector observables S1c and S2c. These S1c and S2c signals can be combined to form a 'reconstructed' energy as $E_{\text {rec }}=$ $W\left(\mathrm{~S} 1 \mathrm{c} / g_{1}+\mathrm{S} 2 \mathrm{c} / g_{2}\right)$ where $W$ is the LXe work function, and $g_{1}$ and $g_{2}$ are the S1c and S2c signal gains as mentioned in Sec. II. The spectra of this new $E_{\text {rec }}$ quantity thereby include the effects of detector resolution and detector threshold. Figure 3 shows signal-model spectra in this $E_{\text {rec }}$ quantity as solid lines. Each $E_{\text {rec }}$ signal spectrum is plotted using an amplitude corresponding to its specific rate sensitivity (90\% C.L. exclusion) as calculated in Sec. VII A. Finally, in each panel, the total ER background spectrum (in the $E_{\text {rec }}$ quantity) is included for comparison. The right-hand axis reports the predicted event counts per $\mathrm{keV}$ in the total expected LZ exposure (1000 live days operation).

\section{A. Electromagnetic properties of solar neutrinos: effective magnetic moment and millicharge}

Neutrinos are expected to exhibit nonzero electromagnetic couplings through loop contributions. Such electromagnetic properties include an effective charge (or millicharge) $q_{\nu}$ and an effective magnetic moment $\mu_{\nu}$. In the Standard Model, minimally extended to include neutrino mass, the neutrino magnetic moment scales proportionally to neutrino mass as 

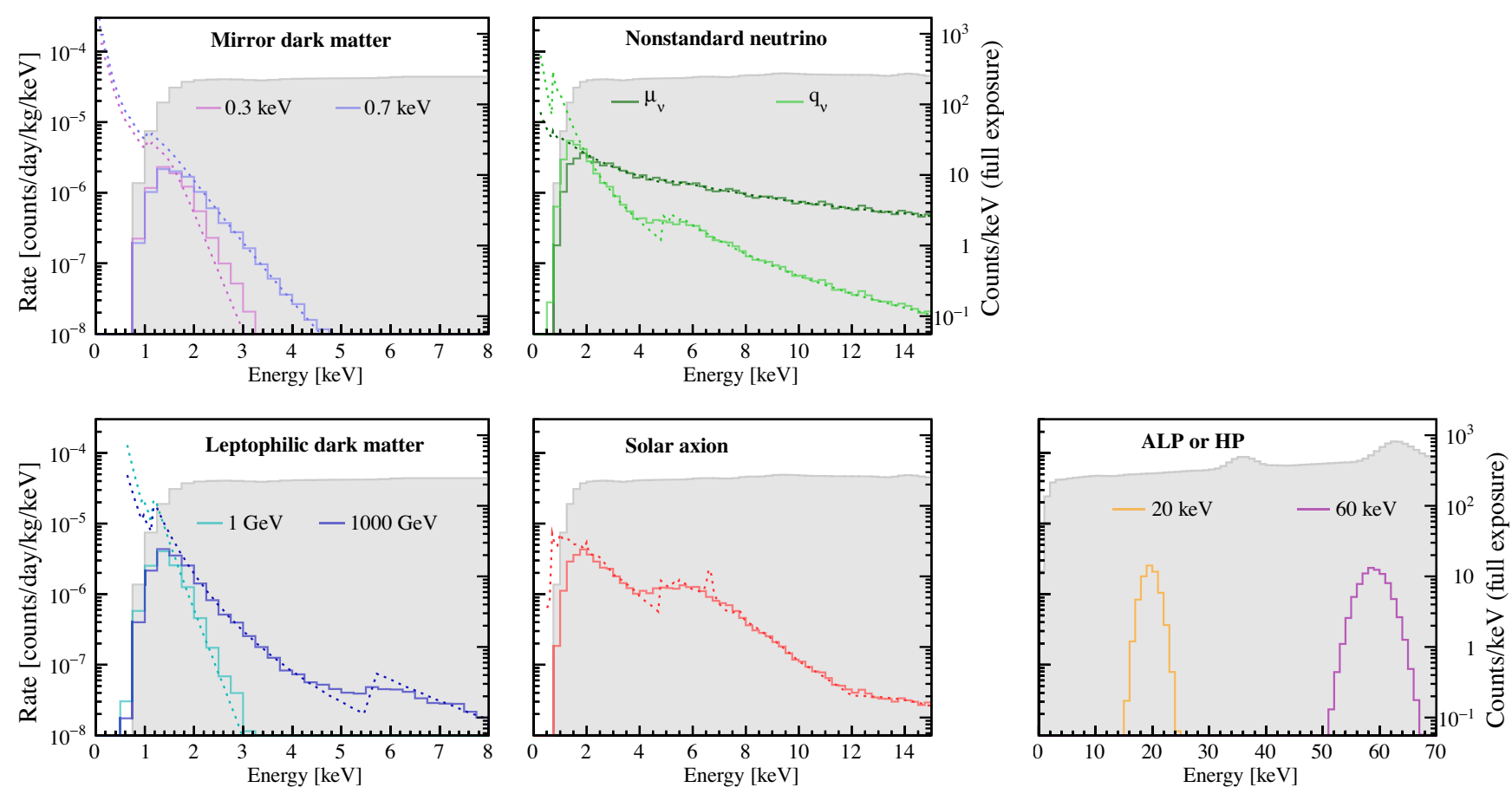

FIG. 3. ER signal spectra studied in this work. True deposited energies are shown using a dotted line and reconstructed energies that include smearing and threshold effects with solid lines. The expected background, also including experimental effects, is shown as the gray region. Each signal spectrum is scaled to the $90 \%$ C.L. rejection sensitivity, as described in Secs. VI and VII. The right vertical axis specifies counts/keV in the $5.6 \mathrm{t} 1000 \mathrm{~d}$ exposure, and is intended to give the reader a sense of the expected statistical fluctuations.

$\mu_{\nu} \approx 3.2 \times 10^{-19}\left(m_{\nu} / 1 \mathrm{eV}\right) \mu_{\mathrm{B}}$, where $m_{\nu}$ is a neutrino mass, $\mu_{\mathrm{B}}=\frac{e h}{4 \pi m_{e}}$ is the Bohr magneton, and $\mu_{\nu}$ is the neutrino magnetic moment of that mass state [38]. Electromagnetic interactions at these small scales are beyond current experimental reach, but searches for a neutrino magnetic moment and millicharge serve as useful tests of physics beyond the Standard Model. Many extensions predict significant enhancement to neutrino electromagnetic properties [39-41]. In the case that the neutrino is a Dirac particle, it is difficult to enhance the electromagnetic properties without a proportional increase to the neutrino mass, which is already tightly constrained. This relationship with mass is less necessary in the Majorana case, and so any observation of neutrino electromagnetic properties would be a strong indicator of the neutrino being a Majorana particle.

Interactions via a millicharge or magnetic moment add terms to the total neutrino-electron scattering cross section, as

$$
\begin{aligned}
\left(\frac{d \sigma_{\nu, e}}{d T_{e}}\right) \simeq & \left(\frac{d \sigma_{\nu, e}}{d T_{e}}\right)_{\text {weak }}+\frac{\pi \alpha^{2}}{m_{e}^{2}}\left(\frac{1}{T_{e}}-\frac{1}{E_{\nu}}\right)\left(\frac{\mu_{\nu}}{\mu_{\mathrm{B}}}\right)^{2} \\
& +\frac{2 \pi \alpha}{m_{e}}\left(\frac{1}{T_{e}^{2}}\right) q_{\nu}^{2}
\end{aligned}
$$

where $\alpha$ is the fine structure constant, $m_{e}$ is the electron mass, and $T_{e}$ can be taken as the energy of the recoiling electron. In the energy regime relevant to most experimental sensitivities, $T_{e} \ll E_{\nu}$, meaning the magnetic moment and millicharge scattering rates fall as $T_{e}^{-1}$ and $T_{e}^{-2}$, respectively.

Equation (1) is a simplified description, assuming that the electron is unbound. Atomic binding effects can be thought of, to first order, as reducing the number of electrons available for very low-energy scatters, thereby reducing the effective total scattering cross section and scattering rate at low energies. The full calculation of atomic effects requires the RRPA method mentioned in Sec. IV, specific to the target atomic structure. This calculation has recently been performed for neutrino electromagnetic scattering on Xe atoms by Hsieh et al. [42], and it is these calculated spectra which serve as the starting point for our work, shown as the dashed lines in the upper middle panel of Fig. 3. One can see both the overall $T_{e}^{-1}$ and $T_{e}^{-2}$ scaling, and the slight steps at the energies of the Xe electron shells.

Existing experimental sensitivities to neutrino electromagnetic properties vary by neutrino source and flavor mixture. Reactor neutrinos fluxes, composed of pure $\bar{\nu}_{e}$, have been used by the GEMMA and TEXONO experiments to set upper limits of $\mu_{\bar{\nu}_{e}}<2.9 \times 10^{-11} \mu_{B}$ and $q_{\bar{\nu}_{e}}<$ $1.5 \times 10^{-12} e_{0}$, where $e_{0}$ is the positron charge $[43,44]$. Solar neutrinos $\left(\nu_{\text {solar }}\right)$ arrive in terrestrial detectors as an incoherent mixture of the three flavor states, and 
electromagnetic interaction sensitivities can be quoted for the effective properties of that flavor mixture. Panda-X-II has recently set an upper limit on the magnetic moment of $\mu_{\nu_{\text {solar }}}<4.9 \times 10^{-11} \mu_{\mathrm{B}}$ [45], while Borexino earlier set a stronger upper limit of $\mu_{\nu_{\text {solar }}}<2.8 \times 10^{-11} \mu_{\mathrm{B}}$ [46]. XMASS-I has set an upper limit on the millicharge of $q_{\nu_{\text {solar }}}<5.4 \times 10^{-12} e_{0}$ [47]. XENON1T has recently observed evidence of an excess that can be interpreted as a positive observation of $\mu_{\nu_{\text {solar }}} \in(1.4,2.9) \times 10^{-11} \mu_{\mathrm{B}}$ [32]. ${ }^{1}$ This same XENON1T data could similarly be interpreted as a positive observation of $q_{\nu_{\text {solar }}} \in(1.7,2.3) \times$ $10^{-12} e_{0}$ [48].

As in other solar neutrino experiments, LZ will expose a massive low-background target to the solar neutrino flux. As can be seen in Fig. 3, while the Standard Model solar neutrino recoils are not expected to dominate the rate, the sharply falling spectral shape that arises in the case of an electromagnetic enhancement, coupled to the $\sim 1 \mathrm{keV}$ threshold, allows xenon detectors to compete with the much larger masses of dedicated neutrino observatories such as BOREXINO. This trade-off between exposure and threshold is described in Ref. [49].

\section{B. Solar axions}

The strong $C P$ problem relates to the unnaturally small value of the $C P$ violating term in $\mathrm{QCD}$. The Standard Model can again be extended to provide a solution, for example, the Peccei-Quinn mechanism [50] introduces an additional $U(1)$ chiral symmetry that is spontaneously broken. The $C P$ violating term is then replaced by a dynamical field that automatically goes to zero by minimizing the potential. Axions are the hypothetical particle resulting from the field; Nambu-Goldstone bosons from the spontaneous breaking of this symmetry at some scale $f_{a}$. Couplings of axions to leptons, hadrons and photons are inversely proportional to $f_{a}$ and the smallest values $\left(f_{a} \leq 10^{-9} \mathrm{GeV}\right)$ have been ruled out by experimental searches [51]. Axion mass is also inversely proportional to $f_{a}$; therefore axions are expected to be very light and very weakly interacting. Production by a nonthermal realignment mechanism [52-54] can generate sufficient quantities of cosmologically stable collisionless axions to make them a viable dark matter candidate. Further production in stellar environments is also possible, leading to emission from stars.

Axion-electron interactions would occur via the axioelectric effect, which is analogous to the photoelectric effect. This could produce ERs in LZ from solar axions

\footnotetext{
${ }^{1}$ The XENON1T result emphasizes an important caveat, that if an unconstrained ${ }^{3} \mathrm{H}$ background component is included, the statistical significance of the $\mu_{\nu_{\text {solar }}}$ evidence decreases from $3.2 \sigma$ to $0.9 \sigma$.
}

emitted by the Sun. The solar axion flux, resulting from production of axions by reactions in the solar plasma, depends on the coupling of axions to electrons (coupling constant $\left.g_{\mathrm{Ae}}\right)$, photons $\left(g_{\mathrm{Ag}}\right)$, and nucleons $\left(g_{\mathrm{An}}\right)$. We consider three solar axion production mechanisms associated with these couplings:

(1) Axion-electron coupling: Atomic, Bremsstrahlung, and Compton $(\mathrm{ABC})$ [55].

(2) Axion-nucleon coupling: ${ }^{57} \mathrm{Fe}$ deexcitation [56].

(3) Axion-photon coupling: Primakoff effect [57].

The relative importance of these production mechanisms depends on the axion model. In hadronic models, such as KSVZ [58,59], the axion has no tree-level coupling to hadrons or leptons, so the Primakoff production dominates. However, for general axion models, such as DFSZ [60,61], the ABC processes, due to the electron coupling, will dominate production. Here no model is assumed and the three components are treated independently of one another.

The $\mathrm{ABC}$ reactions, driven by the axion-electron coupling, comprise of atomic axiorecombination and axiodeexcitation (A), electron ion and electron electron bremsstrahlung (B), and Compton scattering (C). Despite A having the largest cross section it only contributes for metal ions, which are less abundant in the Sun than hydrogen, helium, and electrons; therefore B dominates. The flux is found by integrating the sum of emission rates from $\mathrm{ABC}$, multiplied by the phase space density, over the volume of the Sun-tabulated values for this flux are given in Ref. [55].

The axion-nucleon coupling can lead to axion emission from M1 transitions as a result of deexcitation from thermally excited nuclides within the Sun. This requires an accessible first excited state with an M1 transition to the ground state, in addition to having a high natural abundance in the Sun. The ${ }^{57} \mathrm{Fe}$ isotope fulfils these conditions; it has a $14.4 \mathrm{keV}$ first excited state that may be populated given the solar temperature $\sim 1.3 \mathrm{keV}$, and has a $2.8 \times 10^{-8} \%$ solar abundance by mass fraction. The state is known to deexcitate via M1 transitions to the ground state, emitting $\gamma$-rays and internal conversion electrons. Deexcitation through the emission of a $14.4 \mathrm{keV}$ axion would produce a flux [62]

$$
\Phi_{a}=\left(\frac{k_{a}}{k_{\gamma}}\right)^{3} \times\left(g_{\mathrm{An}}^{\text {eff }}\right)^{2}\left[4.56 \times 10^{23} \mathrm{~cm}^{-2} \mathrm{~s}^{-1}\right]
$$

where $k_{a}$ and $k_{\gamma}$ are the momenta of the axion and photon, and $g_{\mathrm{An}}^{\text {eff }}$ is the effective axion nucleon coupling.

The axion-photon coupling drives Primakoff production-particles with a two-photon vertex can be produced from thermal photons in an external field. The strong magnetic fields and thermal photons in the Sun enable this production mechanism, with flux [57] 
$\frac{d \Phi}{d E}=g_{10}^{2} E 2.481 e^{-E / 1.205}\left[6 \times 10^{10} \mathrm{~cm}^{-2} \mathrm{~s}^{-1} \mathrm{keV}^{-1}\right]$,

where $g_{10}=g_{A \gamma} / 10^{-10} \mathrm{GeV}^{-1}$.

All of these flux components must be multiplied by the axioelectric cross section and the xenon atomic number density in order to find the expected rate in LXe. The solid line in the lower middle panel of Fig. 3 shows the expected energy spectrum in $E_{\text {rec }}$ for the ABC component. Event rates are again dominated by the near-threshold behavior and the experimental resolution smooths out atomic shell effects.

\section{Axionlike particles}

Axionlike particles (ALPs) are a general type of massless Nambu-Goldstone boson or massive pseudo NambuGoldstone boson that appears in many BSM models as a result of the spontaneous breaking of additional (and/or approximate) global symmetries. Although they share some qualitative properties, couplings of generic ALPs to Standard Model particles are far less constrained than for axions, i.e., ALP masses and their coupling to photons are not related and ALPs are not linked to the Peccei-Quinn mechanism in QCD. Thus, while axions acquire mass from mixing with neutral pseudoscalar mesons $\left(m_{\mathrm{A}} f_{\mathrm{A}} \sim m_{\pi} f_{\pi}\right)$, ALPs do not, i.e., the quantity $m_{\mathrm{ALP}} f_{\mathrm{ALP}}$ is less restricted than for QCD axions. This makes the parameter space for ALPs much wider in the experimental context.

Absorption of an ALP by a bound electron is analogous to the ordinary photoelectric effect, with the photon energy $\omega$ replaced by the ALP rest mass $m_{\mathrm{ALP}}$. The cross section of an ALP absorption (i.e., the axioelectric effect) can be expressed in terms of the photoelectric cross section $\sigma_{\mathrm{PE}}$ $\left(\omega=m_{\mathrm{ALP}}\right)$ as [63]

$$
\frac{\sigma_{\mathrm{ALP}} v}{\sigma_{\mathrm{PE}}\left(\omega=m_{\mathrm{ALP}}\right) c} \approx \frac{3 m_{\mathrm{ALP}}^{2}}{4 \pi \alpha f_{\alpha}^{2}},
$$

where $f_{\alpha} \equiv 2 m_{\mathrm{e}} / g_{\mathrm{Ae}}$ and $g_{\mathrm{Ae}}$ are the dimensional and dimensionless coupling constants respectively. $m_{\mathrm{ALP}}$ is the mass and $v \sim 10^{-3} c$ is the velocity of the ALP Cold Dark Matter. The expected event rate $\left(\mathrm{kg}^{-1} \mathrm{day}^{-1}\right)$ in the detector is [63]

$$
R_{\mathrm{ALP}} \simeq \frac{1.2 \times 10^{19}}{A} g_{\mathrm{Ae}}^{2} \sigma_{\mathrm{PE}} m_{\mathrm{ALP}}
$$

where $\sigma_{\mathrm{PE}}$ is expressed in barns and $m_{\mathrm{ALP}}$ in $\mathrm{keV} / \mathrm{c}^{2}$. The rate $R_{\mathrm{ALP}}$ is independent of the velocity $v$, similar to that for the hidden photoelectric effect discussed in Sec. V D.

In the axioelectric effect, the entire ALP rest mass is converted into energy and absorbed by the atomic electron, i.e., the energy deposition is essentially equal to the incoming mass in $\mathrm{keV} / \mathrm{c}^{2}$. The expected energy deposition spectrum in the detector is a monoenergetic peak at the value of the incident mass (then smeared by the experimental resolution, as described in Sec. III). The mass range scanned over thus defines an equivalent range of energy deposition in the detector. In this analysis, the lowest ALP mass considered is $2.0 \mathrm{keV}$, limited by the ER energy threshold of $\sim 1 \mathrm{keV}$. The largest ALP mass considered here is $70 \mathrm{keV}$, though in principle there is no reason this range could not be extended to higher masses in a later analysis. Two example ALP signal models (for $m_{\mathrm{ALP}}=20 \mathrm{keV}$ and $m_{\mathrm{ALP}}=60 \mathrm{keV}$ ) are shown in the lower right plot in Fig. 3.

\section{Hidden/dark photons}

The hidden (or dark) photon (HP) is a hypothetical $U(1)^{\prime}$ gauge boson that resides in a hidden sector, i.e., a sector that does not interact with Standard Model particles through known Standard Model forces. Extra hidden $U(1)^{\prime}$ symmetries often appear in supersymmetric extensions of the Standard Model and in string theories. Hidden photons can obtain a mass either via a hidden Higgs or a Stückelberg mechanism, and interact with the visible sector via loop-induced kinetic mixing [64] with Standard Model hypercharge $U(1)_{Y}$ gauge bosons. If nonthermally produced via the misalignment mechanism in the early Universe they can reproduce the present day dark matter relic abundance [65]. As for the absorption of ALPs, the absorption of a HP by a bound electron is analogous to the ordinary photoelectric effect, with the photon energy $\omega$ replaced by the HP rest mass $m_{\mathrm{HP}}$. The two cross sections, $\sigma_{\mathrm{HP}}$ and $\sigma_{\mathrm{PE}}$, are related by [63]

$$
\frac{\sigma_{\mathrm{HP}} v}{\sigma_{\mathrm{PE}}\left(\omega=m_{\mathrm{HP}}\right) c} \approx \frac{\alpha^{\prime}}{\alpha}
$$

where $v$ is the velocity of the HP CDM particle, $\alpha$ is the electromagnetic fine structure constant and $\alpha^{\prime}$ is its analog for $\mathrm{HP}$

$$
\alpha=\frac{e^{2}}{4 \pi}, \quad \alpha^{\prime}=\frac{g_{\mathrm{h}}^{2}}{4 \pi},
$$

where $e$ and $g_{\mathrm{h}}$ are the visible and gauge couplings, respectively. The kinetic mixing parameter $\kappa$ is related to the fine structure constants $\alpha$ and $\alpha^{\prime}$ as [66]

$$
\kappa=\left(\frac{\alpha^{\prime}}{\alpha}\right)^{1 / 2}
$$

For HPs to constitute the entirety of the typically assumed local cold dark matter they must have a density of $0.3 \mathrm{GeV} / \mathrm{cm}^{3}$ and be nonrelativistic. This corresponds to $v \sim 10^{-3} c$, and the expected interaction rate $\left(\mathrm{kg}^{-1} \mathrm{day}^{-1}\right)$ in the detector in this approximation follows Ref. [63] 


$$
R_{\mathrm{HP}} \simeq \frac{4 \times 10^{23}}{A} \frac{\alpha^{\prime}}{\alpha} \frac{\sigma_{\mathrm{PE}}}{m_{\mathrm{HP}}}
$$

where $\sigma_{\mathrm{PE}}$ is expressed in barns and $m_{\mathrm{HP}}$ in $\mathrm{keV} / \mathrm{c}^{2} . A=$ 131.3 is the atomic mass of $\mathrm{Xe}$. The event rates are independent of the HP velocity distribution in the galactic halo, suggesting the absence of any annual modulation by the Earth's motion, at least to an experimentally relevant level [63].

As for the ALP case (Sec. V C), the expected energy deposition spectrum in the detector should be a monoenergetic peak centered at the value of the incident HP mass and smeared by the experimental resolution. Example energy deposition spectra for $20 \mathrm{keV} / \mathrm{c}^{2}$ and $60 \mathrm{keV} / \mathrm{c}^{2}$ HP or ALP are shown in the lower right plot of Fig. 3again at the amplitudes of projected 90\% C.L. rejection sensitivity according to the profile likelihood ratio tests of Secs. VI and VII.

\section{E. Mirror dark matter}

Whilst hidden photons (discussed above) are a general feature of any hidden sector model with a $U(1)^{\prime}$ group, there are also models where the hidden sector has a specific structure. An example is the mirror dark matter (MDM) model, where the hidden sector is isomorphic to the SM [67]. This symmetry means the hidden sector contains a mirror partner of each Standard Model particle, with the same masses, lifetimes, and self-interactions. The Lagrangian for the system contains the Standard Model Lagrangian, a mirror analog, and a term describing mixing/ portal interactions between the two,

$$
\mathcal{L}_{\text {mix }}=\frac{\varepsilon}{2} F^{\mu \nu} F_{\mu \nu}^{\prime}+\lambda \phi^{\dagger} \phi \phi^{\prime \dagger} \phi^{\prime}
$$

The first term describes kinetic mixing of $U(1)_{Y}$ and mirror $U(1)_{Y}^{\prime}$ and the second describes Higgs $(\phi)$-mirror Higgs $\left(\phi^{\prime}\right)$ mixing. Of interest here is the kinetic mixing interaction with strength $\varepsilon$ [68], which induces a tiny $\varepsilon e$ electromagnetic charge in the mirror particles charged under $U(1)^{\prime}$.

MDM would exist as a multicomponent plasma halo with the main components being mirror electrons, $\mathrm{e}^{\prime}$, and mirror helium nuclei, $\mathrm{He}^{\prime}$. Kinetic mixing between mirror and Standard Model particles would allow mirror electrons in the halo to scatter off $\mathrm{Xe}$ atomic electrons in the LZ detector, inducing ERs.

The rate of ERs depends on the kinetic mixing strength $\varepsilon$ and the local mirror electron temperature $T$. There is also a non-negligible terrestrial effect from the capture and subsequent shielding of incoming mirror electrons [69]. This alters the incoming flux and velocity distributions, as calculated in Ref. [70].
The expected rate is given by [69],

$$
\begin{aligned}
\frac{d R}{d E_{R}}= & g_{T} N_{T} n_{e^{\prime}}^{0} \frac{\lambda}{v_{c}^{0} E_{R}^{2}}\left[1+A_{v} \cos \omega\left(t-t_{0}\right)\right. \\
& \left.+A_{\theta}(\theta-\bar{\theta})\right],
\end{aligned}
$$

where $N_{T}$ is the number of target electrons and $n_{e^{\prime}}^{0}$ is the number density of mirror electrons arriving at the detector. The effective number of free electrons, $g_{T}$, is the number of electrons per target atom with atomic binding energy $\left(E_{b}\right)$ less than the recoil energy $\left(E_{R}\right)$, modeled as a step function for the atomic shells in xenon. The energy-dependent $v_{c}^{0}$ term describes the modified velocity distribution due to terrestrial effects.

Significant modulation, both annual and sidereal, is expected for plasma dark matter models, including MDM. The $A_{v}$ term describes annual modulation due to a Galilean boost from the variation in speed of the Earth with respect to the MDM halo, with $A_{v}$ the amplitude, $\omega$ the angular frequency, $t$ the day for which the rate is calculated, and $t_{0}$ the day with maximum speed with respect to the halo (day 152, June 2nd). The $A_{\theta}$ term describes annual and sidereal modulation due to variation of the angle between the Earth's spin axis and the incoming dark matter wind; $A_{\theta}$ is the amplitude and $\theta(t)$ is the angle between the halo wind and the zenith, with $\bar{\theta}$ being the average over a year.

These date dependent modulation terms are calculated for a given date, with maximum daily variations in rate of approximately $60 \%$ on the 7th June and 8th December. These variations average out over the course of a year and for 1000 live days we find a maximum variation of $7 \%$. However there may be greater variation depending on any gaps in data taking. Since the exact dates are not known yet, a factor of 1.0 is used for these two terms in the rate calculations.

The resulting theoretical energy spectra and corresponding reconstructed energy spectra are shown in the upper left panel of Fig. 3 for two mirror electron temperatures with the kinetic-mixing parameter at the sensitivity level determined in Sec. VII A.

\section{F. Leptophilic dark matter}

Leptophilic dark matter is an appealing alternative to the WIMP hypothesis as it relaxes existing constraints on the parameter space from direct detection and collider searches [71]. We consider one such model, in which the dark matter particle couples exclusively to leptons via the exchange of a heavy mediator in a contact interaction. In this case, the signal of interest to LZ is generated by dark matter scattering off bound electrons, such that the recoil energy acquired by the electron is sufficient to liberate it from its host atom. At typical dark matter velocities, the energy of an electron initially at rest is of the order of a few $\mathrm{eV}$ and 
therefore far below the detection threshold; however, an electron bound to an atom can have a non-negligible momentum, resulting in detectable energy depositions [72].

We calculate the differential event rate using Eq. 6 in Ref. [73]. To compute the atomic excitation factors for xenon, we employ the relativistic Hartree-Fock method as described in Ref. [74] using the AMPSCI package [75]. This treatment is necessary as relativistic effects have been shown to dominate the ionization cross section $[74,76]$. The lower left plot in Fig. 3 shows the expected differential event rate in LZ, with the dominant contribution to the signal arising from scattering off electrons in the 3 s shell.

\section{STATISTICAL ANALYSIS}

We employ frequentist hypothesis tests based on the PLR method, and follow the prescription of Ref. [77] to determine LZs sensitivity to exclude or observe parameters of the models described above.

The comparison between the signal and background is conducted using two-dimensional probability distribution functions (PDFs) in the variables $\mathrm{S} 1 \mathrm{c}$ and $\log (\mathrm{S} 2 \mathrm{c})$. The choice to use 2D PDFs in these observables offers some small advantages in sensitivity compared to the simpler analysis using $E_{\text {rec }}$. The PDFs are produced in NEST using the energy spectra of the signal and backgrounds as in Fig. 3. An extended unbinned likelihood function is then generated with Gaussian-constrained nuisance parameters for the dominant background rates.

The projected exclusion sensitivity reported for a given signal model is the median $90 \%$ C.L. upper limit on the model's strength parameter of interest that would be obtained in repeated background-only experiments. We use a two-sided PLR test statistic [78,79], the signal and background distributions of which are obtained through toy Monte Carlo (MC) experiments generated from the likelihood model (as opposed to relying on asymptotic approximations of the distribution). A total of 5000 toy experiments are used to build the test-statistic distributions under both the null and alternate hypothesis for each parameter of interest value scanned.

Exclusion sensitivities are calculated for all signal models described in Sec. V. Additionally, for those signal models deemed most relevant to the excess reported by XENON1T [32] we also calculate the median parameter of interest values at which the background-only model is rejected at $3 \sigma$ ( $p$-value threshold $\left.1.3 \times 10^{-3}\right)$, indicating evidence of a signal [79]. These selected models are: the solar neutrino magnetic moment, solar neutrino millicharge, solar axions, and the monoenergetic signals from ALPs and HPs. In the ALP and HP sensitivities, the signal energy is varied, and the $3 \sigma$ sensitivity is quoted in a local sense at each energy (without application of the lookelsewhere effect). This philosophy allows the results to be applied for scenarios in which, for example, LZ is serving
TABLE I. We list the expected mean counts for each background component in a $5.6 \mathrm{t} 1000 \mathrm{~d}$ exposure for the specified ranges of energy and S1c. Counts are quoted after the application of single scatter, veto, and fiducial volume selections. The last column specifies the uncertainty (labelled "Unc.") in rate we assume when projecting expected sensitivity in Sec. VI. Solar neutrino-electron scattering is included without uncertainty. Nuclear recoil backgrounds are included in the analysis (see [18]) but are omitted from this table due to their negligible rates within the ER signal region.

\begin{tabular}{|c|c|c|c|c|c|}
\hline \multicolumn{6}{|c|}{ Expected counts in $5.6 \mathrm{t} 1000 \mathrm{~d}$} \\
\hline \multicolumn{3}{|c|}{ Component Energy range $[\mathrm{keV}]$} & \multicolumn{2}{|c|}{ S1c range [phd] } & \multirow{2}{*}{$\frac{\text { Unc }}{(\%)}$} \\
\hline & $1.5-10$ & $1.5-70$ & $0-100$ & $0-570$ & \\
\hline${ }^{222} \mathrm{Rn}$ & 1216 & 9873 & 2504 & 11998 & \\
\hline${ }^{220} \mathrm{Rn}$ & 171 & 1394 & 353 & 1694 & 24 \\
\hline${ }^{85} \mathrm{Kr}$ & 45 & 378 & 93 & 462 & $\ldots$ \\
\hline${ }^{136} \mathrm{Xe}$ & 166 & 8796 & 603 & 13186 & 50 \\
\hline${ }^{124} \mathrm{Xe}$ & 38 & 3287 & 56 & 3299 & 30 \\
\hline Solar $\nu$ & 336 & 2418 & 670 & 2845 & \\
\hline Det.+Surf.+Env. $\gamma$ & 93 & 754 & 191 & 916 & 20 \\
\hline TOTAL & 2065 & 26900 & 4470 & 34400 & \\
\hline
\end{tabular}

to search for a signal possibly observed in another experiment.

The expected rates and uncertainties for the background components used in the likelihood are listed in Table I, following [18]. In this analysis, signals from decays of ${ }^{85} \mathrm{Kr}$, ${ }^{222} \mathrm{Rn}$, and ${ }^{220} \mathrm{Rn}$ (and importantly, their progeny ${ }^{214} \mathrm{~Pb}$ and ${ }^{212} \mathrm{~Pb}$ ) are grouped together as a single background component with a single, combined rate of uncertainty due to their similar spectral shapes in the region of interest. Two background components are included without rate uncertainties, and their normalizations are fixed in the analysis. The first is solar neutrino-electron recoils, the rate of which is dominated by neutrinos from the $p p$ chain. The flux uncertainty is $\sim 0.6 \%$ [80] and the scattering rate uncertainty is $2 \%-3 \%$ [29], both subdominant to other uncertainties. The second is the general category of NR backgrounds, due to the negligible number of which are expected in the ER signal region in $\{\mathrm{S} 1 \mathrm{c}, \log (\mathrm{S} 2 \mathrm{c})\}$ space, as mentioned previously. In the solar-axion analysis, the Primakoff component of the signal model was used as an additional, unconstrained nuisance parameter that floats in the fit.

As mentioned in Sec. IV, we include two unexpected but possible backgrounds in the specific case of projecting sensitivity to $3 \sigma$ evidence: ${ }^{37} \mathrm{Ar}$ and ${ }^{3} \mathrm{H}$. In a real experimental dataset, evidence for new physics would not be claimed if the observed excess were similarly consistent with some 'unexpected but possible' background contamination. Because they are unexpected, these two backgrounds are not included in the generation of toy MC data, but because they are possible, they are included in the likelihood fits to those data. In a real experiment, some 
externally-derived constraint on ${ }^{37} \mathrm{Ar}$ and ${ }^{3} \mathrm{H}$ concentrations or rates may be applied, but in this work we conservatively apply no such constraint. The result of this method is that no level of statistical evidence is possible for signals with spectral shape identical to that of either ${ }^{37} \mathrm{Ar}$ or ${ }^{3} \mathrm{H}$, and sensitivity is reduced for any signal with sufficiently high overlap with either of these two backgrounds.

\section{RESULTS}

\section{A. Sensitivity projections}

We summarize the results of the sensitivity projections, starting with the case of neutrino electromagnetic properties. We find that the full LZ exposure will be capable of rejecting solar-neutrino magnetic moments $\mu_{\nu_{\text {solar }}}$ greater than $6.2 \times 10^{-12} \mu_{\mathrm{B}}$ and millicharges $q_{\nu_{\text {solar }}}$ greater than $1.4 \times 10^{-13} e_{0}$, both at $90 \%$ C.L. Sensitivity to $3 \sigma$ significance is found to be $9.5 \times 10^{-12} \mu_{\mathrm{B}}$ and $1.9 \times 10^{-13} e_{0}$. To reemphasize, these sensitivities to evidence conservatively include unconstrained rates of the unexpected ${ }^{37} \mathrm{Ar}$ and ${ }^{3} \mathrm{H}$ backgrounds, weakening sensitivity.

These sensitivities are shown in Fig. 4(a), together with existing experimental constraints on solar neutrino properties. Constraints using pure $\bar{\nu}_{e}$ neutrinos (from reactor-based experiments) are also shown. XENON1T has seen evidence for an excess in rate at low energies consistent with several signal hypotheses including a neutrino magnetic moment [32]. The 90\% C.L. allowed region under this magnetic moment interpretation is indicated. We see from the LZ discovery and exclusion sensitivities that the XENON1T excess, under the magnetic moment hypothesis, will be robustly tested by LZ. We note that various astrophysical constraints are stronger than these direct experimental constraints, see for example a summary in [41]. Given the sharply falling spectral shape, particularly in the millicharge case, an S2-only treatment of the LZ data may have a similar or stronger sensitivity to these signals, but the backgrounds to such searches are difficult to estimate a priori and the topic is beyond the scope of this work.

For solar axions we find projected $90 \%$ C.L. exclusion sensitivity to axioelectric coupling $g_{\mathrm{Ae}}$ values as low as $1.58 \times 10^{-12}$, and $3 \sigma$ evidence for discovery for $g_{\mathrm{Ae}}$ equal to $1.84 \times 10^{-11}$. These sensitivity projections are shown in Fig. 4(c). This shows an improvement over previous experimental results, including a factor $\sim 2.5$ in exclusion sensitivity over the LUX result [81]. The most stringent limit at present is the indirect constraint derived from the observed brightness of the tip of the Red Giant branch [82], which in turn places limits on the allowed additional cooling by axion emission. The indirect constraint from the solar neutrino flux [83] and experimental constraints from both solid state [84-87] and LXe direct detection [45,88-90] experiments are also presented. Also shown in Fig. 4(c) is a constraint from a model that includes a stellar basin of gravitationally bound axions, giving a second component of the flux [91]. This result is obtained by recasting previous experimental limits, but there is a large uncertainty from calculation of the ejection time of particles from the solar system. The XENON1T excess [32] can be interpreted as an allowed range of $g_{\mathrm{Ae}}$ for the case of other couplings (e.g., $g_{\mathrm{A} \gamma}$ ) being small, and that allowed region in $g_{\mathrm{Ae}}$ is shown in Fig. 4(c). LZ will robustly test a solar axion interpretation of the XENON1T excess.

A scan over ALP masses $\left(2-70 \mathrm{keV} / \mathrm{c}^{2}\right)$ constrains the expected $g_{\mathrm{Ae}}$ to be no larger than $\sim 7.6 \times 10^{-14}$ (at $\sim 64.3 \mathrm{keV}$, the energy of the ${ }^{124} \mathrm{Xe} \mathrm{KK}$ peak). LZ is expected to give a limit approximately two orders of magnitude more stringent than existing results [92-95]. A scan over HP masses $\left(2-70 \mathrm{keV} / \mathrm{c}^{2}\right)$ constrains $\kappa^{2}$ to be no larger than $\sim 7.4 \times 10^{-28}$ (at $\sim 64.3 \mathrm{keV}$ ). While at intermediate energies $(\sim 8-30 \mathrm{keV})$ the indirect (Red Giant) limit on $\kappa^{2}$ is still the most stringent, $\mathrm{LZ}$ is expected to give a better limit at lower $(\leq 8 \mathrm{keV})$ and higher energies, i.e., $\sim(30-70) \mathrm{keV}$. An improvement of approximately two orders of magnitude over the existing direct-detection results [92-95] is expected. Because an unconstrained ${ }^{37} \mathrm{Ar}$ component is included in projections of sensitivity to $3 \sigma$ evidence, we expect no sensitivity to monoenergetic signals at the $2.8 \mathrm{keV}$ energy of the ${ }^{37} \mathrm{Ar}$ decay. Note the discontinuity at this energy in both the ALP and HP $3 \sigma$-evidence sensitivity curves (dashed red curves in Figs. 4(d) and 4(b)).

The projected exclusion sensitivity to mirror dark matter kinetic mixing for various local mirror electron temperatures is shown in Fig. 4(e). This shows that, in the event of no signal, we would be able to exclude mirror electron temperatures down to $0.25 \mathrm{keV}$ for this model, giving an improvement over the first direct detection search carried out by LUX [70].

The projected exclusion sensitivity to the DM-electron scattering cross section is as low as $5.8 \times 10^{-41} \mathrm{~cm}^{2}$ for a dark matter particle of mass $2 \mathrm{GeV} / \mathrm{c}^{2}$, as shown in Fig. 4(f). This represents an improvement of nearly three orders of magnitude over the strongest direct detection limits to date, calculated using S2-only data from XENON10 and XENON100 in Ref. [96]. An S2-only analysis of LZ data has the potential to extend this sensitivity to lower masses.

\section{B. Effect of intrinsic beta backgrounds}

Intrinsic Xe contaminants constitute the dominant ER background in LZ at low energies, largely due to $\beta$ decays from the ${ }^{212} \mathrm{~Pb}\left({ }^{214} \mathrm{~Pb}\right)$ progenies of ${ }^{220} \mathrm{Rn}\left({ }^{222} \mathrm{Rn}\right)$. These isotopes are especially troublesome as their decays may result in beta particles with no accompanying radiation, either because the beta decay was directly to the ground state, or the associated gamma rays escape without detection. The rate of this dominant background depends strongly on the success of dust-reducing cleanliness protocols during detector assembly and with the somewhat uncertain radon emanation of components in the cold xenon environment. It is thus relevant to investigate how 


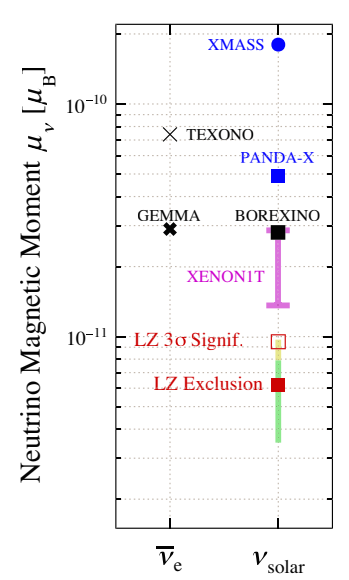

Neutrino Type

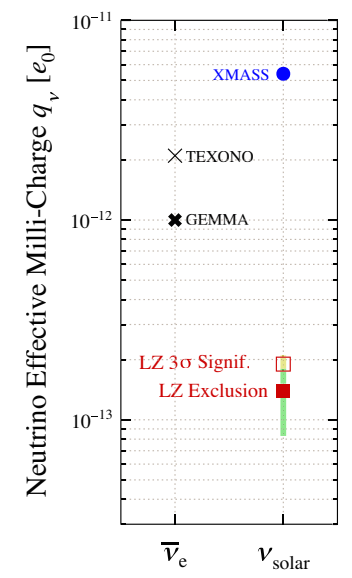

Neutrino Type

(a)

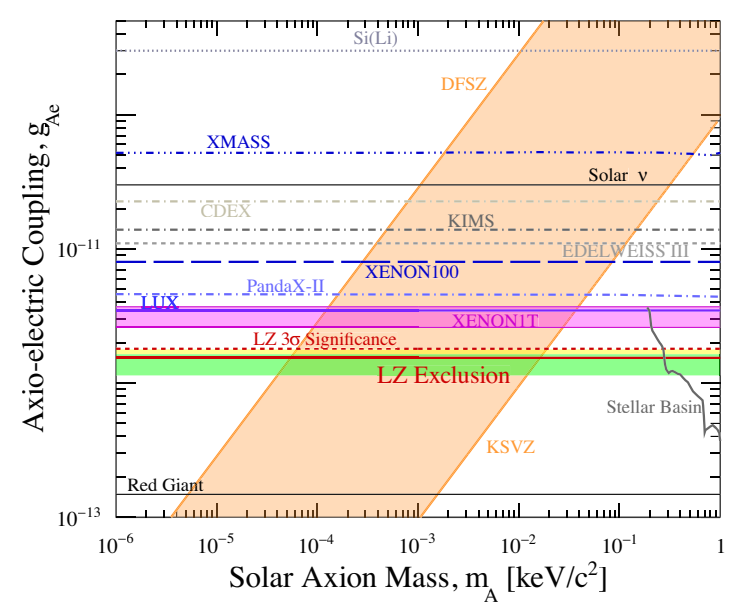

(c)

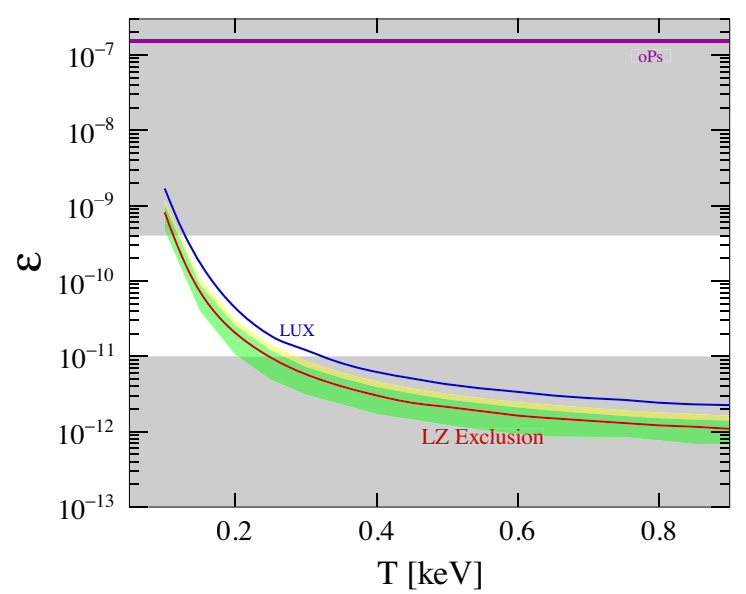

(e)

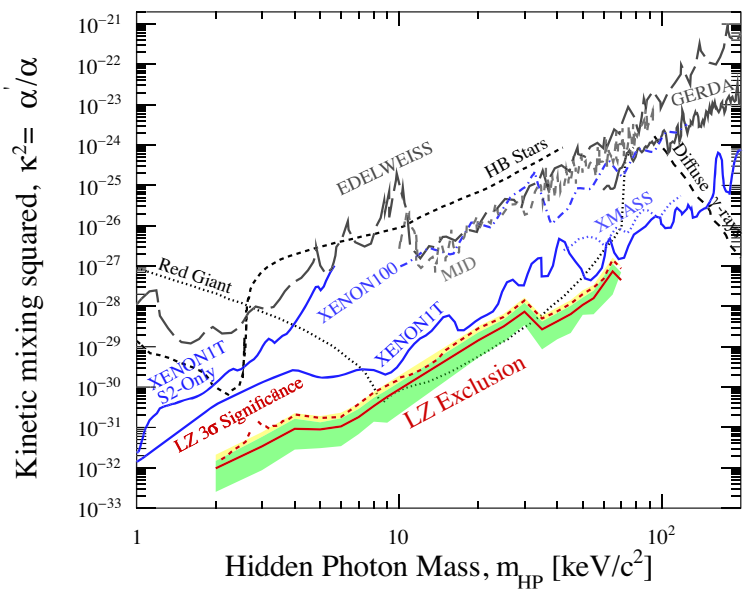

(b)

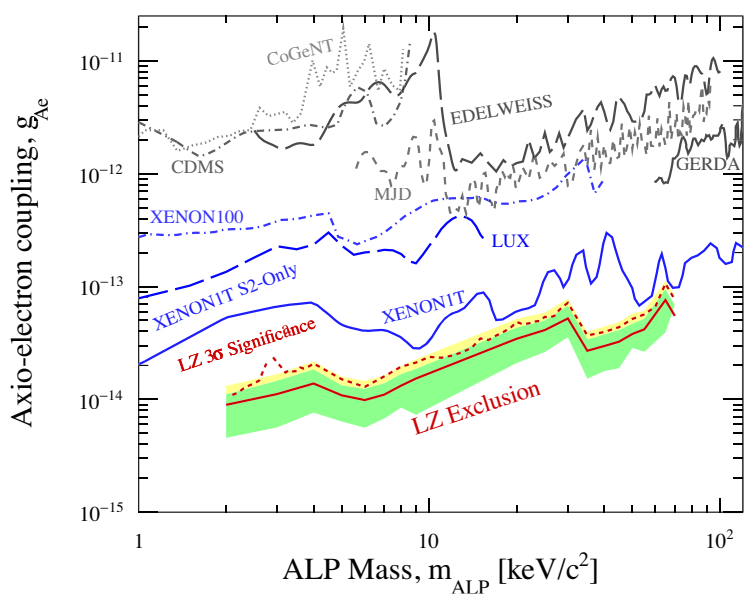

(d)

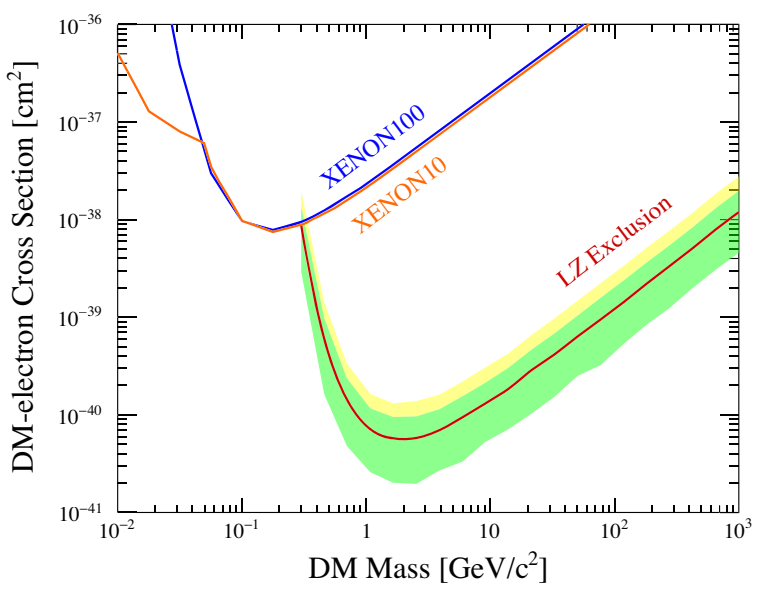

(f)

FIG. 4. Projected $90 \%$ C.L. exclusion sensitivity to (a) electromagnetic neutrino couplings $\mu_{\nu}$ and $q_{\nu}$, (b) kinetic mixing squared, $\kappa^{2}$ for hidden photons, (c) axioelectric coupling for solar axions, (d) axio-electric coupling for galactic ALPs, (e) mirror dark matter kinetic mixing, and (f) DM-electron scattering cross section for leptophilic dark matter. $\pm 1 \sigma$ (green) and $+2 \sigma$ (yellow) bands are also shown. For selected models, sensitivity to $3 \sigma$ evidence is also shown (labeled 'LZ $3 \sigma$ Significance'). Results from other experiments and astrophysical constraints are referenced in the text. 
signal sensitivity varies under differing radon contamination scenarios. As in the sensitivity projections using the baseline background model (see Table I), three backgrounds of uniform spatial distribution and similar (nearly flat) spectral shape are grouped together as a single background component: ${ }^{222} \mathrm{Rn}+{ }^{220} \mathrm{Rn}+{ }^{85} \mathrm{Kr}$. We now vary this grouped 'distributed betas' component by a factor of 10 greater and smaller than the baseline expectation (keeping the rate uncertainty nuisance parameter proportional at 24\%) and repeat selected exclusion sensitivity projections under these varied background assumptions. We show the results of these studies in Fig. 5, plotting projected 90\% CL exclusion sensitivity as a function of the dominating ${ }^{222} \mathrm{Rn}$ portion of the varied background component.

Given the 1000-day exposure time assumed, any possible signal competes with a large number of background counts, typically numbering in the hundreds of events per $\mathrm{keV}$. In this high-stats regime, sensitivity to signal counts

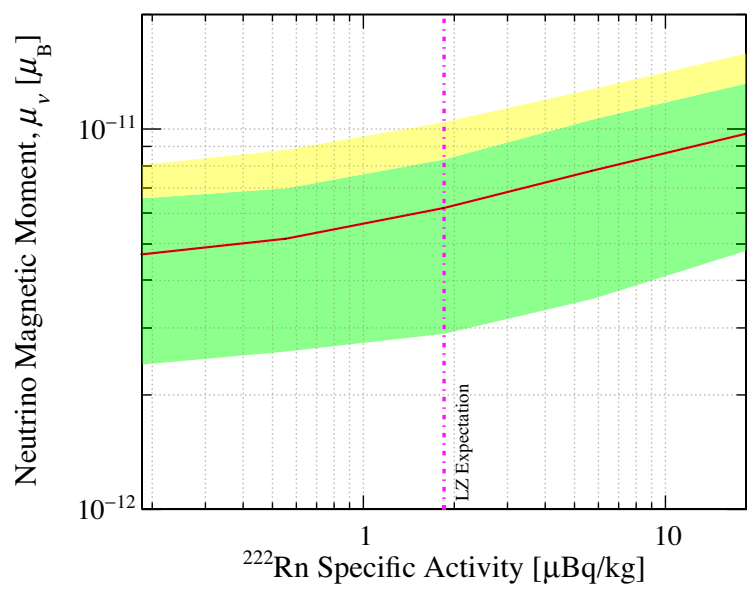

(a)

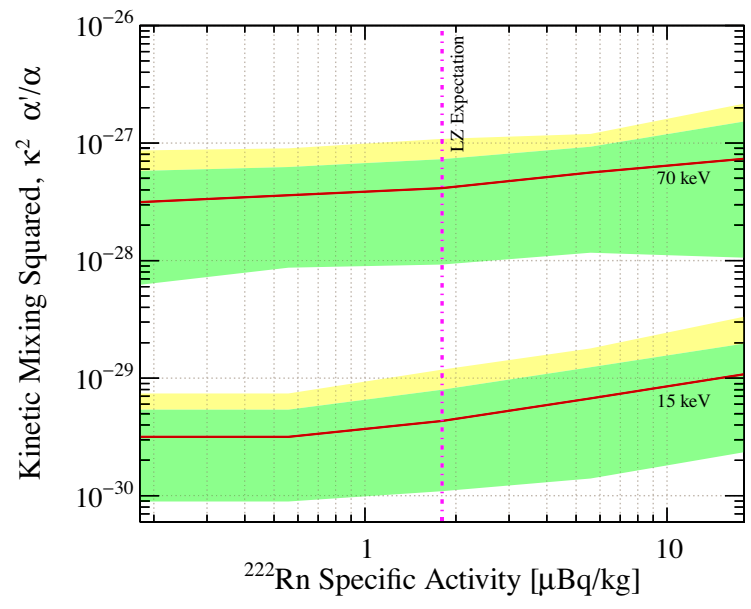

(c) changes in proportion to the square root of the background counts in the relevant signal region. The PLR studies under varied background expectation confirm this expectation at low energies when ${ }^{222} \mathrm{Rn}$ forms the dominant background. This square root scaling is seen to weaken in two regimes: first, when the ${ }^{222} \mathrm{Rn}$ is reduced by a significant factor (such that the solar neutrino scattering rate begins to dominate), or second, when the signal model is constrained to higher energies, above $\sim 40 \mathrm{keV}$, such that the $\nu \nu \beta \beta$ decay of ${ }^{136} \mathrm{Xe}$ begins to dominate. The effect of solar neutrino backgrounds can be seen most clearly as a flattening in the left-most portion of Fig. 5(b), and the effect of ${ }^{136} \mathrm{Xe} \nu \nu \beta \beta$ can be seen as a difference in slope between the $15 \mathrm{keV}$ and $70 \mathrm{keV}$ HP sensitivities.

As mentioned previously, the shapes of the beta decay spectra that dominate the low-energy background have recently been subject to new theoretical calculation. This results in a suppression of their rates, at energies relevant to

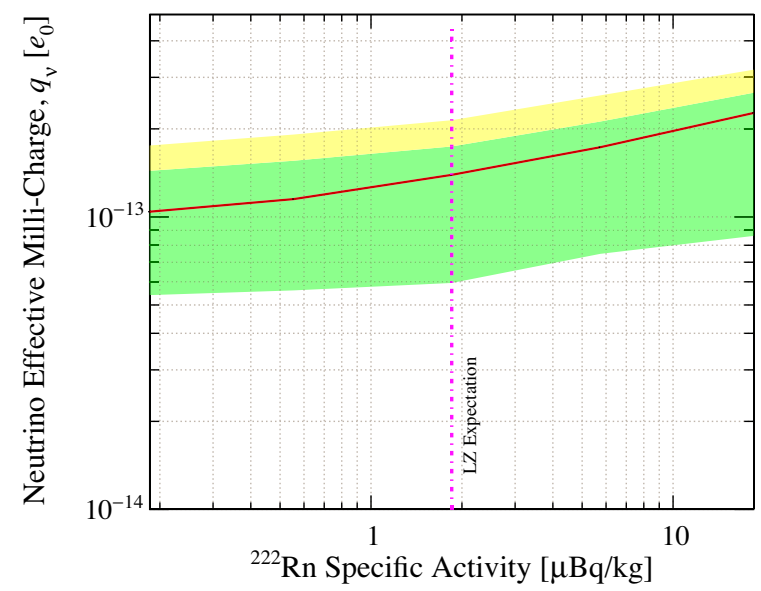

(b)

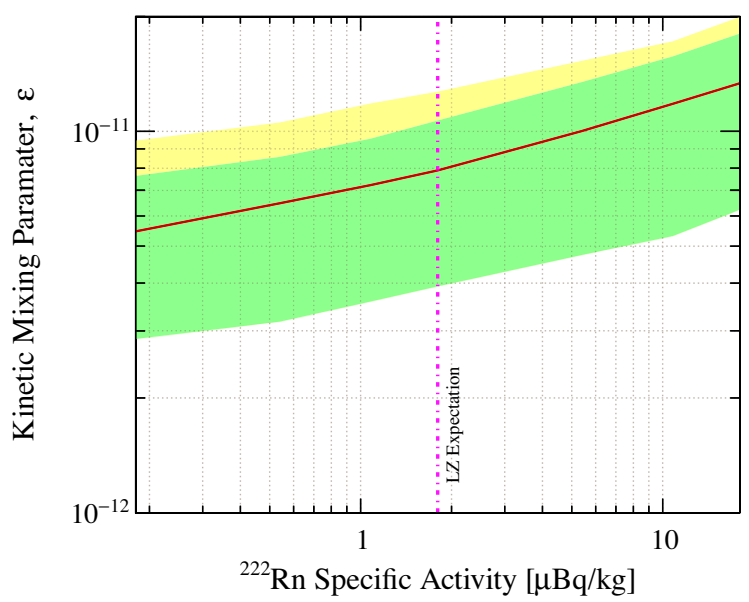

(d)

FIG. 5. Variation of $90 \%$ C.L. exclusion sensitivity to (a) solar neutrino magnetic moment, (b) solar neutrino effective millicharge, (c) kinetic mixing squared, $\kappa^{2}$ for 15 and $70 \mathrm{keV} / c^{2}$ hidden photons, and (d) mirror dark matter kinetic mixing for local mirror electron temperature $0.3 \mathrm{keV}$. $\pm 1 \sigma$ (green) and $+2 \sigma$ (yellow) bands are also shown. 
this work, by $\sim 19 \%$ for the dominant ${ }^{214} \mathrm{~Pb}$ species, and smaller amounts for the other subdominant species ${ }^{212} \mathrm{~Pb}$ and ${ }^{85} \mathrm{Kr}$ [36]. The effect of a $\sim 19 \%$ reduction in lowenergy background beta decay rate equates to shifting the LZ expectation line in Fig. 5(b) to the left, from $1.8 \mu \mathrm{Bq} / \mathrm{kg}$ to $1.46 \mu \mathrm{Bq} / \mathrm{kg}$. It can be seen therefore that these refinements in spectral shape change the projected LZ sensitivities of this work by less than $10 \%$.

The main conclusion from these studies is that LZ sensitivity to these signals will remain world leading under a variety of reasonable background assumptions.

\section{CONCLUSION}

There are a number of well-motivated extensions to the Standard Model of particle physics that the LZ experiment will be able to test with unprecedented sensitivity. Here we have presented the sensitivity of LZ to theoretical models in which either new particles, such as axions, or new mechanisms of interaction, such as enhanced loopinduced effective electromagnetic neutrino properties, result in additional low-energy ERs. In total seven models are considered, covering a range of signal shape profile and energy. In each model, LZ is projected to have worldleading sensitivity. In particular, LZ will thoroughly test any new physics explanation of the recent XENON1T excess. LZ is currently being commissioned and will commence data taking at SURF in 2021.

\section{ACKNOWLEDGMENTS}

The research supporting this work took place in whole or in part at the Sanford Underground Research Facility (SURF) in Lead, South Dakota. Funding for this work is supported by the U.S. Department of Energy, Office of Science, Office of High Energy Physics under Contracts No. DE-AC02-05CH11231, No. DE-SC0020216, no. DE-SC0012704, No. DE-SC0010010, No. DE-AC0207CH11359, No. DE-SC0012161, No. DE-SC0014223,
No. DE-SC0010813, No. DE-SC0009999, No. DENA0003180, No. DE-SC0011702, No. DESC0010072, No. DE-SC0015708, No. DE-SC0006605, No. DESC0008475, No. DE-FG02-10ER46709, No. UW PRJ82AJ, No. DE-SC0013542, No. DE-AC0276SF00515, No. DE-SC0018982, No. DE-SC0019066, No. DE-SC0015535, No. DE-SC0019193 No. DE-AC5207NA27344, and No. DOE-SC0012447. This research was also supported by U.S. National Science Foundation (NSF); the United Kingdom Science and Technology Facilities Council under Grants No. ST/M003655/1, No. ST/ M003981/1, No. ST/M003744/1, No. ST/M003639/1, No. ST/M003604/1, No. ST/R003181/1, No. ST/ M003469/1, No. ST/S000739/1, No. ST/S000666/1, No. ST/S000828/1, No. ST/S000879/1, No. ST/S000933/ 1, No. ST/S000747/1, No. ST/S000801/1 and No. ST/ R003181/1 (JD); Portuguese Foundation for Science and Technology (FCT) under Grant No. PTDC/FIS-PAR/28567/ 2017; the Institute for Basic Science, Korea (Budget No. IBS-R016-D1). We acknowledge additional support from the STFC Boulby Underground Laboratory in the United Kingdom the GridPP [97,98] and IRIS Consortium, in particular at Imperial College London and additional support by the University College London Cosmoparticle Initiative. This research used resources of the National Energy Research Scientific Computing Center, a D. O.E. Office of Science User Facility supported by the Office of Science of the U.S. Department of Energy under Contract No. DE-AC02-05CH11231. This work was completed in part with resources provided by the University of Massachusetts' Green High Performance Computing Cluster. The University of Edinburgh is a charitable body, registered in Scotland, with the Registration No. SC005336. The assistance of SURF and its personnel in providing physical access and general logistical and technical support is acknowledged. We thank Jiunn-Wei Chen and his group for handing off numerical results from their RRPA calculations, and we thank Patrick Draper for useful discussions.
[1] K. Lesko, Phys. Procedia 61, 542 (2015).

[2] G.J. Alner et al. (ZEPLIN-I Collaboration), Astropart Phys. 23, 444 (2005).

[3] G. J. Alner et al. (ZEPLIN-II Collaboration), Astropart. Phys. 28, 287 (2007).

[4] D. Yu. Akimov et al. (ZEPLIN-III Collaboration), Phys. Lett. B 709, 14 (2012).

[5] D. S. Akerib et al. (LUX Collaboration), Phys. Rev. Lett. 118, 021303 (2017).

[6] J. Angle et al. (XENON10 Collaboration), Phys. Rev. Lett. 100, 021303 (2008).
[7] E. Aprile et al. (XENON100 Collaboration), Phys. Rev. D 94, 122001 (2016).

[8] E. Aprile et al. (XENON1T Collaboration), Phys. Rev. Lett. 121, 111302 (2018).

[9] M. Xiao et al. (PANDAX-I Collaboration), Sci. China Phys. Mech. Astron. 57, 2024 (2014).

[10] A. Tan et al. (PANDAX-II Collaboration), Phys. Rev. Lett. 117, 121303 (2016).

[11] D. S. Akerib et al. (LUX-ZEPLIN Collaboration), J. Astropart. Phys. 125, 102480 (2021). 
[12] D. S. Akerib et al. (LUX-ZEPLIN), Astropart. Phys. 96, 1 (2017).

[13] D. S. Akerib et al. (LUX Collaboration), Phys. Rev. D 93, 072009 (2016).

[14] E. Aprile et al. (XENON100 Collaboration), Phys. Rev. D 97, 092007 (2018).

[15] B Yan et al. (PandaX-II Collaboration), Chin. Phys. C 45, 075001 (2021).

[16] B. J. Mount et al. (LUX-ZEPLIN Collaboration), arXiv: 1703.09144.

[17] D. Akerib et al. (LUX-ZEPLIN Collaboration), Nucl. Instrum. Methods Phys. Res., Sect. A 953, 163047 (2020).

[18] D. S. Akerib et al. (LUX-ZEPLIN Collaboration), Phys. Rev. D 101, 052002 (2020).

[19] D. S. Akerib et al. (LUX-ZEPLIN Collaboration), arXiv: 2101.08753.

[20] D. S. Akerib et al. (LUX-ZEPLIN Collaboration), Phys. Rev. C 102, 014602 (2020).

[21] D.S. Akerib et al. (LUX-ZEPLIN Collaboration), Eur. Phys. J. C 80, 1044 (2020).

[22] C. H. Faham, V. M. Gehman, A. Currie, A. Dobi, P. Sorensen, and R. J. Gaitskell, J. Instrum. 10, P09010 (2015).

[23] D. S. Akerib et al. (LUX Collaboration), Phys. Rev. D 97, 102008 (2018).

[24] D. S. Akerib et al. (LUX Collaboration), Phys. Rev. D 101, 042001 (2020).

[25] S. Agostinelli et al. (GEANT4 Collaboration), Nucl. Instrum. Methods Phys. Res., Sect. A 506, 250 (2003).

[26] M. Szydagis, N. Barry, K. Kazkaz, J. Mock, D. Stolp, M. Sweany, M. Tripathi, S. Uvarov, N. Walsh, and M. Woods (NEST Collaboration), J. Instrum. 6, P10002 (2011).

[27] M. Szydagis et al. (NEST Collaboration) (2018), Noble Element Simulation Technique v2.0 online.

[28] A. Nilima, Ph.D thesis, University of Edinburgh, 2020, https://era.ed.ac.uk/handle/1842/37516.

[29] J.-W Chen, H.-C Chi, C.-P. Liu, and C. P. Wu, Phys. Lett. B 774, 656 (2017).

[30] V. I. Kopeikin, L. A. Mikaelyan, V. V. Sinev, and S. A. Fayans, Phys. At. Nucl. 60, 1859 (1997).

[31] E. Aprile et al. (XENON-1T Collaboration), Nature (London) 568, 532 (2019).

[32] E. Aprile et al. (XENON1T Collaboration), Phys. Rev. D 102, 072004 (2020).

[33] M. Szydagis, C. Levy, G. M. Blockinger, A. Kamaha, N. Parveen, and G. R. C. Rischbieter, Phys. Rev. D 103, 012002 (2021).

[34] A. E. Robinson, arXiv:2006.13278.

[35] D. Akerib et al. (LUX-ZEPLIN Collaboration) (to be published).

[36] S. Haselschwardt, J. Kostensalo, X. Mougeot, and J. Suhonen, Phys. Rev. C 102, 065501 (2020).

[37] J. Kotila and F. Iachello, Phys. Rev. C 85, 034316 (2012).

[38] K. Fujikawa and R. E. Shrock, Phys. Rev. Lett. 45, 963 (1980).

[39] N. F. Bell, V. Cirigliano, M. J. Ramsey-Musolf, P. Vogel, and M. B. Wise, Phys. Rev. Lett. 95, 151802 (2005).

[40] N. F. Bell, M. Gorchtein, M. J. Ramsey-Musolf, P. Vogel, and P. Wang, Phys. Lett. B 642, 377 (2006).
[41] C. Giunti and A. Studenikin, Rev. Mod. Phys. 87, 531 (2015).

[42] C.-C. Hsieh, L. Singh, C. P. Wu, J.-W. Chen, H.-C. Chi, C.-P. Liu, M. K. Pandey, and H. T. Wong, Phys. Rev. D 100, 073001 (2019).

[43] A. G. Beda, V. B. Brudanin, V. G. Egorov, D. V. Medvedev, V. S. Pogosov, M. V. Shirchenko, A. S. Starostin (GEMMA Collaboration), Adv. High Energy Phys. 2012, 350150 (2012).

[44] M. Deniz et al. (TEXONO Collaboration), Phys. Rev. D 81, 072001 (2010).

[45] X. Zhou et al. (PandaX Collaboration), Chin. Phys. Lett. 38, 011301 (2021).

[46] M. Agostini et al. (Borexino Collaboration), Phys. Rev. D 96, 091103 (2017).

[47] K. Abe et al. (XMASS Collaboration), Phys. Lett. B 809, 135741 (2020).

[48] A. N. Khan, Phys. Lett. B 809, 135782 (2020).

[49] R. Harnik, J. Kopp, and P. A. N Machado, J. Cosmol. Astropart. Phys. 07 (2012) 026.

[50] R. D. Peccei and H. R. Quinn, Phys. Rev. Lett. 38, 1440 (1977).

[51] J.E. Kim and G. Carosi, Rev. Mod. Phys. 82, 557 (2010).

[52] J. Preskill, M. B. Wise, and F. Wilczek, Phys. Lett. 120B, 127 (1983).

[53] L. F. Abbott and P. Sikivie, Phys. Lett. 120B, 133 (1983).

[54] M. Dine and W. Fischler, Phys. Lett. 120B, 137 (1983).

[55] J. Redondo, J. Cosmol. Astropart. Phys. 12 (2013) 08.

[56] S. Moriyama, Nucl. Phys. B 72, 183 (1999).

[57] M. Kuster, B. Beltrán, and G. G. Raffelt, Axions : Theory, Cosmology, and Experimental Searches (Springer New York, 2008), https://www.worldcat.org/title/axions-theorycosmology-and-experimental-searches/oclc/190776609.

[58] J. Kim, Phys. Rev. Lett. 43, 103 (1979).

[59] M. Shifman, A. Vainshtein, and V. Zakharov, Nucl. Phys. B166, 493 (1980).

[60] M. Dine, W. Fischler, and M. Sredenicki, Phys. Lett. 104B, 199 (1981).

[61] A. Zhitnitsky, Sov. J. Nucl. Phys. 31, 260 (1980).

[62] CAST Collaboration, J. Cosmol. Astropart. Phys. 12 (2009) 002.

[63] M. Pospelov, A. Ritz, and M. Voloshin, Phys. Rev. D 78, 115012 (2008).

[64] S. A. Abel, M. D. Goodsell, J. Jaeckel, V. V. Khoze, and A. Ringwald, J. High Energy Phys. 07 (2008) 124.

[65] P. Arias, D. Cadamuro, M. Goodsell, J. Jaeckel, J. Redondo, and A. Ringwald, J. Cosmol. Astropart. Phys. 06 (2012) 013.

[66] F. Brummer and J. Jaeckel, Phys. Lett. B 675, 360 (2009).

[67] R. Foot, Int. J. Mod. Phys. A 29, 1430013 (2014).

[68] R. Foot, H. Lew, and R. Volkas, Phys. Lett. B 272, 67 (1991).

[69] R. Foot, Phys. Lett. B 789, 592 (2019).

[70] D. S. Akerib et al. (LUX Collaboration), Phys. Rev. D 101, 012003 (2020).

[71] N. F. Bell, Y. Cai, R. K. Leane, and A. D. Medina, Phys. Rev. D 90, 035027 (2014).

[72] J. Kopp, V. Niro, T. Schwetz, and J. Zupan, Phys. Rev. D 80, 083502 (2009). 
[73] B. M. Roberts and V. V. Flambaum, Phys. Rev. D 100, 063017 (2019).

[74] B. M. Roberts, V. A. Dzuba, V. V. Flambaum, M. Pospelov, and Y. V. Stadnik, Phys. Rev. D 93, 115037 (2016).

[75] B. M. Roberts (2020), https://github.com/benroberts999/ ampsci.

[76] B. M. Roberts, V. V. Flambaum, and G. F. Gribakin, Phys. Rev. Lett. 116, 023201 (2016).

[77] W. A. Rolke, A. M. Lopez, and J. Conrad, Nucl. Instrum. Methods Phys. Res., Sect. A 551, 493 (2005).

[78] G. Cowan, K. Cranmer, E. Gross, and O. Vitells, Eur. Phys. J. C 71, 1554 (2011); 73, 2501(E) (2013).

[79] D. Baxter, I. M. Bloch, E. Bodnia, and X. Chen, Eur. Phys. J. C arXiv:2105.00599.

[80] N. Vinyoles et al. Astrophys. J. 835, 202 (2017).

[81] D. S. Akerib et al. (LUX Collaboration), Phys. Rev. Lett. 118, 261301 (2017).

[82] O. Straniero, C. Pallanca, E. Dalessandro, I. Dominguez, F. R. Ferraro, M. Giannotti, A. Mirizzi, and L. Piersanti, Astron. Astrophys. 644, A166 (2020).

[83] P. Gondolo and G. G. Raffelt, Phys. Rev. D 79, 107301 (2009).

[84] A. V. Derbin, I. S. Drachnev, A. S. Kayunov, and V. N. Muratova, JETP Lett. 95, 339 (2012).

[85] E. Armengaud et al. (EDELWEISS Collaboration), Phys. Rev. D 98, 082004 (2018).
[86] Y.S. Yoon et al. (KIMS Collaboration), J. High Energy Phys. 06 (2016) 011.

[87] Y. Wang et al. (CDEX Collaboration), Phys. Rev. D 101, 052003 (2020).

[88] K Abe et al. (XMASS Collaboration), Phys. Lett. B 724, 46 (2013).

[89] E. Aprile et al. (XENON100 Collaboration), Phys. Rev. D 90, 062009 (2014).

[90] C. Fu et al. (PANDAX Collaboration), Phys. Rev. Lett. 119, 181806 (2017).

[91] K. Van Tilburg, Phys. Rev. D 104, 023019 (2021).

[92] K. Abe et al. (XMASS Collaboration), Phys. Lett. B 787, 153 (2018).

[93] E. Aprile et al. (XENON-1T Collaboration), Phys. Rev. Lett. 123, 251801 (2019).

[94] H. An, M. Pospelov, J. Pradler, and A. Ritz, Phys. Rev. D 102, 115022 (2020).

[95] M. Agostini et al. (GERDA Collaboration), Phys. Rev. Lett. 125, 011801 (2020).

[96] R. Essig, T. Volansky, and T.-T. Yu, Phys. Rev. D 96, 043017 (2017).

[97] P. J. W. Faulkner, L. S. Lowe, C. L. A. Tan et al., J. Phys. G 32, N1 (2005).

[98] D. Britton, A. Cass, P. Clarke et al., Phil. Trans. R. Soc. A 367, 2447 (2009). 